\title{
Top-down control of hippocampal signal-to-noise by prefrontal long-range inhibition
}

4 Department of Psychiatry, UCSF Weill Institute for Neurosciences, Kavli Institute for

5 Fundamental Neuroscience, Center for Integrative Neuroscience, University of California San

6 Francisco, San Francisco, California

$7 \quad$ * Corresponding author: vikaas.sohal@ucsf.edu 


\section{Summary}

9 The prefrontal cortex (PFC) is postulated to exert 'top-down control' by modulating information

10 processing throughout the brain to promote specific actions based on current goals. However, the

11 pathways mediating top-down control remain poorly understood. In particular, knowledge about

12 direct prefrontal connections that might facilitate top-down prefrontal control of information

13 processing in the hippocampus remains sparse. Here we describe novel monosynaptic long-range

14 GABAergic projections from PFC to hippocampus. These preferentially inhibit vasoactive

15 intestinal polypeptide expressing interneurons, which are known to disinhibit hippocampal

16 microcircuits. Indeed, stimulating prefrontal-hippocampal GABAergic projections increases

17 hippocampal feedforward inhibition and reduces hippocampal activity in vivo. The net effect of

18 these actions is to specifically enhance the signal-to-noise ratio for hippocampal representations

19 of objects. Correspondingly, stimulation of PFC-to-hippocampus GABAergic projections

20 promotes object exploration. Together, these results elucidate a novel top-down pathway in

21 which long-range GABAergic projections target disinhibitory microcircuits, thereby enhancing

22 signals and network dynamics underlying exploratory behavior. 


\section{Introduction}

24 The prefrontal cortex (PFC) plays a crucial role in executive functions and the top-down control of brain activity and behavior (Gazzaley and D'Esposito, 2007; Miller and Cohen, 2001; Miller, 2000). It is postulated that PFC bidirectionally communicates with several cortical and subcortical brain regions, monitoring and gating their ongoing activity, in order to exert topdown executive control over behavior. One brain region that is known to bidirectionally interact with the PFC is the hippocampus (HPC), a key brain region for processing spatial information, and using spatial representations to guide behavior. Accumulating evidence in humans and animal models highlights an essential role of network interactions between the PFC and HPC in cognitive and emotional behaviors (Eichenbaum, 2017; Jin and Maren, 2015; Preston and Eichenbaum, 2013; Shin and Jadhav, 2016; Sigurdsson and Duvarci, 2016; Yu and Frank, 2015). Importantly, abnormal PFC-HPC interactions are thought to contribute to cognitive and emotional deficits in several neuropsychiatric disorders, including schizophrenia, depression, and anxiety disorders (Cunniff et al., 2020; Godsil et al., 2013; Kupferschmidt and Gordon, 2018; Li et al., 2015; Sigurdsson et al., 2010). Owing to the importance of PFC-HPC interactions in normal and pathological behaviors, much work has been focused on elucidating how these regions interact.

Functional imaging studies in humans as well as rodent studies using lesions and pharmacological inactivation have shown that concurrent activity in, and communication between, the PFC and HPC is essential for spatial exploratory behaviors (Bähner et al., 2015; Churchwell et al., 2010; DeVito and Eichenbaum, 2010; Floresco et al., 1997; Wang and Cai, 2006; Yoon et al., 2008). Neural activity and network oscillations synchronize across the PFC and HPC during spatial exploratory behaviors (Colgin, 2011; Jones and Wilson, 2005; O'Neill et al., 2013; Spellman et al., 2015). In particular, oscillatory activity in HPC leads PFC activity when rats explore spatial contexts, but this pattern of synchronization switches to PFC leading when rats explore objects in their environment (Place et al., 2016) or arrive at decision points in a maze (Hallock et al., 2016). Furthermore, inactivating the PFC alters the encoding of spatial information in the HPC (Guise and Shapiro, 2017; Kyd and Bilkey, 2003). These findings suggest that the PFC exerts top-down control over HPC activity at key behavioral timepoints, but

52 knowledge about direct anatomical projections that mediate this kind of top-down prefrontal

53 control is lacking. In fact, whereas much is known about the direct anatomical pathways from the

54 HPC-to-PFC (Hoover and Vertes, 2007; Jay and Witter, 1991), most top-down communication

55 in the PFC-to-HPC direction is thought to occur indirectly, via the thalamic nucleus reuniens

56 (NR) (Hoover and Vertes, 2012; Vertes et al., 2007; Xu and Südhof, 2013). Not only are the 57 anatomical substrates for top down control unknown, the manner in which top down control 
58 operates is also unclear. I.e., does the PFC exerts top-down control by transmitting specific

59 information, e.g., representations of specific actions or goals, or alternatively, does it modulate

60 the network state, changing the nature of emergent circuit computations in downstream regions?

61 Previous studies of PFC-HPC interactions have focused on direct and indirect excitatory

62 (glutamatergic) connections between these structures. Growing evidence indicates that cortical

63 circuits also include specialized populations of long-range projecting GABAergic (LRG)

64 inhibitory neurons (Jinno et al., 2007; Melzer and Monyer, 2020; Tamamaki and Tomioka,

65 2010). In some cases, these LRG projections have been shown to control oscillatory

66 synchronization between structures (Christenson Wick et al., 2019; Francavilla et al., 2018;

67 Melzer et al., 2012), suggesting that they may be important regulators of interregional

68 communication. We recently reported that the PFC also contains specialized LRG projection

69 neurons capable of influencing behavior (Lee et al., 2014). Therefore, we hypothesized that a

70 specialized population of PFC LRG projection neurons might serve as the anatomical substrate

71 through which the PFC exerts top-down control over hippocampal information processing.

72 Here, we report a novel population of LRG neurons in the PFC that send direct inhibitory

73 projections to the dorsal hippocampus (dHPC). Notably, these prefrontal LRG projections target

74 local disinhibitory microcircuits and modulate network oscillations in dHPC. Through these

75 actions, PFC-dHPC LRG projections promote network states associated with object exploration,

76 enhance hippocampal representations of object locations, and elicit corresponding increases in

77 the time mice spend exploring objects. Together, our results show how the PFC exerts top-down

78 control over information processing in the HPC by acting through a novel circuit motif: long-

79 range GABAergic projections which inhibit disinhibitory microcircuits, thereby altering

80 emergent network dynamics and promoting specific exploratory behaviors. 


\section{Results}

\section{Hippocampus projecting long-range GABAergic (LRG) neurons in the PFC}

83 To label potential PFC-to-HPC LRG projections, we used Dlxi12b-Cre mice, which specifically

84 express Cre recombinase in GABAergic neurons (Lee et al., 2014; Potter et al., 2009). We injected an adeno-associated virus (AAV) to drive Cre-dependent expression of the fluorescent reporter eYFP (AAV5-EF1 $\alpha$-DIO-eYFP) in the PFC of Dlxi12b-Cre mice (Fig. 1A). After waiting 6-8 weeks for viral transduction, we observed robust eYFP expression in the cell bodies of GABAergic neurons in the PFC and also observed many axonal fibers in the CA1 and dentate gyrus subfields of dHPC (Fig. 1B and Fig. S1). Importantly, no eYFP+ cell bodies were observed in the HPC.

Next, we asked whether these PFC LRG axon terminals synapse onto neurons in the dHPC. To address this, we injected AAV into the PFC of Dlxi12b-Cre mice to drive Credependent expression of Channelrhodopsin-eYFP (ChR2-eYFP) in PFC GABAergic neurons, then, after waiting for expression, made recordings from acute hippocampal slices (Fig. 1C). Notably, optogenetic activation of PFC LRG axonal fibers in dHPC slices elicited robust shortlatency postsynaptic currents (oPSCs) in dHPC neurons. These currents reversed at the GABA reversal potential, were not affected by glutamatergic receptor antagonists, and were completely blocked by bath application of the $\mathrm{GABA}_{\mathrm{A}}$ receptor antagonist gabazine (10 $\left.\mu \mathrm{M}\right)$ (Fig. 1D).

Following the identification of PFC-dHPC LRG projections, we asked whether these dHPC-projecting PFC LRG neurons have distinct electrophysiological and molecular properties, and whether these neurons are located in superficial or deeper cortical layers of the PFC. To address these questions, we used an intersectional strategy to selectively express ChR2-eYFP in dHPC-projecting PFC LRG neurons. Specifically, we injected two viruses: a retrogradely transducing canine adenovirus type-2 Cre (CAV2-Cre) into dHPC, and a Cre-dependent AAV expressing ChR2-eYFP under control of the Dlxi12b enhancer into PFC (Lee et al., 2014) (Fig. 1E, F). We then made ex-vivo patch clamp recordings from dHPC-projecting LRG neurons in

107 PFC (identified by eYFP expression), and recorded reliable short-latency light-evoked action 108 potentials (APs) to confirm that they were ChR2-expressing (Fig. 1G). These recordings revealed 109 that the dHPC-projecting PFC LRG neuronal population is electrophysiologically diverse, 110 comprising neurons with regular spiking (9/16 neurons), irregular spiking (3/16 neurons), and 111 fast spiking (4/16 neurons) physiological properties (Fig. 1H and Table S1). dHPC-projecting 112 PFC LRG neurons were distributed across superficial and deeper layers of the prelimbic (PL) 113 portion of the PFC. By combining injection of a retrograde tracer (Alexa 594-tagged cholera 114 toxin, CTb) in dHPC with immunohistochemistry in PFC (Fig. S2A, B), we found that dHPC115 projecting PFC LRG neurons include parvalbumin (PV), somatostatin (SST), and vasoactive 
116 intestinal polypeptide (VIP)-expressing subpopulations (Fig. S2C, D). We also observed small

117 percentages of calretinin (CR) and neuropeptide-Y (NPY) expressing PFC-dHPC LRG neurons.

118 However, none of the PFC LRG neurons in our study showed immunoreactivity for neuronal

119 nitric oxide synthase (nNOS). Taken together, these results reveal that the dHPC receives direct

120 LRG projections which originate from a heterogeneous population of GABAergic inhibitory

121 neurons located across multiple layers of the PFC.

122

\section{PFC LRG projections target hippocampal disinhibitory interneurons}

124 Next, we asked how electrophysiologically and molecularly heterogeneous PFC LRG projection

125 neurons affect circuit computations in the CA1 subregion, which is the primary output region of

126 the dHPC. Specifically, we asked whether PFC LRG projections target specific cell-types in the

127 CA1 subregion. We expressed ChR2-eYFP in PFC LRG projections and obtained ex-vivo patch

128 clamp recordings from excitatory pyramidal neurons and GABAergic interneurons located in

129 different topographical layers of CA1 subregion in acute hippocampal slices (Fig. 2A).

130 Interestingly, we observed robust optogenetically-evoked IPSCs in CA1 interneurons (55/70

131 connected, henceforth referred to as recipient interneurons), but not in CA1 pyramidal neurons

132 (PNs; 0/38 connected) (details of interneuron and PN classification in Methods). Notably, many

133 of the recipient CA1 interneurons were located near the border between stratum radiatum (SR)

134 and stratum lacunosum-moleculare (SLM) (Fig. 2B). Furthermore, recipient CA1 interneurons

135 comprised physiologically heterogeneous subtypes including regular spiking, irregular spiking,

136 and fast spiking interneurons (Fig. 2B and Table S2). In order to determine whether the PFC

137 LRG projections target molecularly defined interneuron subtypes in CA1, we filled a subset of

138 the recipient interneurons with biocytin and quantified the immunoreactivity for three molecular

139 markers commonly expressed in CA1 interneurons- PV, SST, and VIP. Surprisingly, we found

140 that a majority of recipient interneurons expressed VIP (7/11). By contrast, none of the recipient

141 interneurons we examined expressed PV or SST (0/10) (Fig. 2C).

\section{PFC LRG projections regulate excitatory input integration in CA1 microcircuit}

144 Since VIP is predominantly expressed by interneuron-selective interneurons (ISIs) which 145 produce circuit disinhibition in CA1 (Acsády et al., 1996a; 1996b; Chamberland and Topolnik, 146 2012; Turi et al., 2019), we hypothesized that PFC-dHPC LRG projections may inhibit VIP+ 147 ISIs, thereby reducing disinhibition and increasing feedforward inhibition in the CA1 148 microcircuit. To test this prediction, we quantified the effect of optogenetic stimulation of PFC 149 LRG projections on excitatory and inhibitory postsynaptic potentials (EPSPs and IPSPs) elicited 150 by two major afferent input pathways: Schaffer collateral (SC) and temporoammonic (TA) inputs 151 (Fig. 2D). Specifically, during ex-vivo patch clamp recordings from CA1 PNs, we delivered 
152 electrical stimulation to SC or TA inputs concomitant with optogenetic stimulation of ChR2+

153 PFC-dHPC LRG axon fibers. While the optogenetic stimulation of PFC-dHPC LRG axons

154 alone did not elicit discernable postsynaptic potentials in CA1 PNs, concomitant electrical and 155 optogenetic stimulation significantly increased the size of IPSPs relative to EPSPs for both SC 156 and TA inputs (Fig. 2E, F and Fig. S3A, B). This reduction in the excitation to inhibition ratio 157 (E/I ratio) for SC and TA inputs is consistent with our prediction and suggests that the PFC LRG 158 projections increase feedforward inhibition by inhibiting disinhibitory VIP+ interneurons in 159 CA1.

We then asked whether increased feedforward inhibition during stimulation of PFC LRG projections affects the input-output transformation performed by CA1 PNs. Coincident activation

162 of SC and TA input pathways, often in a theta-burst stimulation (TBS) pattern, is known to cause 163 supralinear input summation and spiking in CA1 PNs (Ang et al., 2005; Bittner et al., 2015; 164 Malik and Johnston, 2017). This nonlinear input integration and coincidence detection in CA1 165 PNs is tightly regulated by the activity of CA1 interneurons and considered crucial for 166 hippocampal information processing (Grienberger et al., 2017; Milstein et al., 2015). To 167 determine how PFC-dHPC LRG projections modulate input integration in CA1 PNs, we 168 combined electrical TBS of SC and TA inputs with optogenetic stimulation of PFC-dHPC LRG 169 projections (20 Hz, $5 \mathrm{~ms}$ pulses) (Fig. 2G). Again, consistent with increased feedforward 170 inhibition, optogenetic stimulation of LRG projections reduced firing and EPSP summation 171 during TBS (Fig. 2H). Importantly, firing of CA1 PNs in response to depolarizing current 172 injections (i.e., neuronal depolarization without recruitment of microcircuit inhibition) was not 173 affected by optogenetic stimulation of these PFC LRG projections (Fig. S3C). Taken together, 174 our ex-vivo electrophysiological analyses show how PFC-dHPC LRG projections regulate 175 synaptic integration and input-output gain by enhancing feedforward inhibition onto CA1 PNs 176 (Fig. S3D).

\section{PFC-dHPC LRG projections promote object exploration}

179 Communication between PFC and HPC is implicated in many spatial and object exploration 180 behaviors (DeVito and Eichenbaum, 2010; Jin and Maren, 2015; Preston and Eichenbaum, 2013; 181 Spellman et al., 2015; Yu and Frank, 2015). Notably, both structures synchronize at theta 182 frequency with dHPC leading when rodents enter a spatial context, but the directionality 183 switches to PFC leading when animals sample an object (Place et al., 2016). This suggests an 184 important role for top-down communication from PFC to dHPC during object exploration. 185 Therefore, we quantified how PFC-dHPC LRG projections affect object exploration in freely 186 behaving mice. Optogenetic stimulation of PFC-dHPC LRG projections (20 Hz, $5 \mathrm{~ms}$ pulses, $187473 \mathrm{~nm}, \sim 3-4 \mathrm{~mW}$ ) dramatically increased the time Dlxil2b-Cre mice spent engaged in novel 
object exploration (NOE) (Fig. 3A, B). Light delivery alone had no effect in control (Crenegative) mice. Increases in NOE occurred during both early and late portions of the testing session (Fig. 3C, D), and reflected increased numbers of both short- and long-duration bouts of object exploration (Fig. 3E). Optogenetic stimulation of PFC-dHPC LRG projections did not affect the distance travelled in an open field, time spent on the stimulated side during a real-time place preference task, or the time spent exploring a novel juvenile mouse (Fig. S4A-C). Thus, activating PFC-dHPC LRG projections specifically increases NOE without nonspecifically affecting movement or other exploratory behaviors.

\section{PFC LRG projections promote network oscillations associated with object exploration}

198 Next, we explored potential circuit mechanisms through which PFC-dHPC LRG projections might impact NOE. We did this in two ways. First, we recorded local field potentials (LFPs) to determine whether stimulation of PFC-dHPC LRG projections might induce network states conducive to NOE (Fig. 4A). In comparison to baseline home cage (HC) exploration, NOE recruited synchronized oscillations in the low-gamma $(25-55 \mathrm{~Hz})$ band across the PFC-dHPC network. Specifically, during NOE we observed a significant increase in low-gamma power in both structures as well as an increase in low-gamma phase synchrony between the PFC and dHPC (Fig. 4B). While the increase in low-gamma activity was most prominent, NOE was also associated with significant increases in power (but not synchrony) for high-gamma activity (both frequency oscillations is particularly notable because previous studies have shown that object exploration increases low-gamma synchrony between hippocampal subfields (Trimper et al., 2017). Since microcircuit interactions between local CA1 interneurons and PNs are known to

211 critically regulate gamma oscillations (Csicsvari et al., 2003; Tukker et al., 2007), we

212 hypothesized that by modulating microcircuit inhibition, PFC-dHPC LRG projections could

213 contribute to NOE-associated changes in gamma activity. To test whether PFC-dHPC LRG

214 projections might support these changes in network activity, we combined optogenetic

215 stimulation with multisite LFP recordings in Dlxi12b-Cre mice expressing ChR2 in PFC-dHPC

216 LRG projections (Fig. 4C). Indeed, optogenetic stimulation of PFC LRG terminals (20 Hz, $5 \mathrm{~ms}$ 217 pulses, $473 \mathrm{~nm}, \sim 3-4 \mathrm{~mW}$ ) in dHPC mimicked the increases in both low-gamma LFP power and 218 low-gamma phase synchrony observed during NOE (Fig. 4D and Fig. S5C, D). Thus, PFC LRG 219 projections promote a network state associated with object exploration, a behavior known to rely 220 on top-down PFC-dHPC communication. 
223 Having established that PFC-dHPC LRG projections promote network states associated with

224 NOE as well as NOE itself, we next studied how these projections affect NOE-associated

225 hippocampal activity at the level of single cells. For this, we expressed jGCaMP7f in dHPC CA1

226 neurons (Dana et al., 2019) and used one-photon miniaturized microscopes (miniscopes) to

227 record in vivo neuronal $\mathrm{Ca}^{2+}$ activity while mice explored novel objects. Concurrently, we

228 expressed the red-shifted excitatory opsin ChrimsonR (Klapoetke et al., 2014; Stamatakis et al.,

229 2018) in PFC-dHPC LRG projections (Fig. 5A-C and Fig. S6A, B). On day 1, mice explored a

230 novel object in the absence of optogenetic stimulation of PFC LRG projections. Across all

231 neurons, $\mathrm{Ca}^{2+}$ activity decreased significantly during NOE, relative to the HC epoch (Fig. 5D),

232 although a small fraction of neurons (13/55 neurons) had higher activity during NOE (Fig. S6C).

233 On day 2, we optogenetically stimulated PFC-dHPC LRG projections during both HC and NOE

234 epochs. Stimulating LRG projections during the HC epoch significantly reduced activity

235 (compared to the pre-stimulation HC period). Activity was then further reduced when the mice

236 subsequently engaged in NOE (Fig. 5D and Fig. S6C). This overall reduction in population

237 activity in vivo is consistent with our ex-vivo observation that activating PFC-dHPC LRG

238 projections tends to enhance feedforward inhibition and reduce spiking in CA1 PNs.

\section{PFC-dHPC LRG projections enhance the encoding of objects by hippocampal ensembles}

241 To assess whether these global changes in CA1 activity were associated with changes in how the

242 hippocampus encodes NOE-relevant information, we compared the NOE-driven changes in 243 neuronal activity on day 1 (no stimulation) vs. day 2 (LRG stimulation). As shown by the 244 seminal discovery of place cells, dHPC CA1 neurons encode information by preferentially firing 245 in specific spatial locations (Moser et al., 2008; O'Keefe, 1976; Wilson and McNaughton, 1993).

246 Therefore, we asked whether the PFC-dHPC LRG projections affect the encoding of object 247 location by individual hippocampal neurons. Specifically, for each neuron, we defined its 'object 248 signal-to-noise ratio' (Object_SNR) as the change in its activity within a zone surrounding the 249 object location before vs. after introducing the object (activity was z-scored relative to the mean 250 and standard deviation outside the object zone) (Fig. 6A). Based on this metric, neurons that 251 increased or decreased activity in the object zone by one standard deviation had Object_SNR of 2521 or -1 , respectively. During light stimulation, the activity of neurons decreased both within and 253 outside of the object zone; the standard deviations of neuronal activity also decreased (Fig. 6B). 254 Depending on exactly how these changes were distributed across neurons, Object_SNR values 255 could potentially increase, decrease, or remain unchanged. In fact, we observed that stimulating 256 PFC LRG projections significantly increased Object_SNR values relative to the no light 257 condition (Fig. 6B, right-most panel). Notably, light delivery alone did not affect the neuronal 258 activity or the Object_SNR in control (opsin-negative) mice (Fig. 6C). Furthermore, in opsin- 
259 expressing mice, LRG stimulation did not affect an analogous 'SNR' calculated for a control 260 zone on the opposite side of the cage (instead of the object zone) (Fig. 6D, E). Thus, even though 261 PFC-dHPC LRG projections potentiate feedforward inhibition and reduce overall network 262 activity, their net effect on hippocampal encoding is to specifically enhance object-driven signals 263 in individual CA1 neurons. 


\section{Discussion}

265 Interactions between the PFC and HPC have been implicated in numerous aspects of cognition

266 and emotion, including decisions about whether to engage in exploratory behaviors. While

267 monosynaptic excitatory projections from the ventral HPC are believed to transmit specific

268 information, e.g., the locations of goals, to the PFC (Spellman et al., 2015; Wang and Cai, 2006),

269 pathways through which the PFC exerts top-down control over the HPC, and the exact nature of

270 these top-down effects, have remained less well understood. Here, we describe a novel

271 monosynaptic projection from the PFC-to-dHPC. There are many unusual features of this

272 projection: it is GABAergic and targets hippocampal VIP+ ISIs, thus representing a 'doubly

273 disinhibitory' long-range motif. We show that this projection modulates microcircuit dynamics

274 in the CA1 region of dHPC, increasing feedforward inhibition, reducing spiking evoked by

275 afferent inputs, and enhancing low-gamma activity that is synchronized between the PFC and

276 dHPC. Furthermore, we show that activation of these projections reduces in vivo activity while

277 specifically enhancing the representations of objects in the dorsal CA1. Lastly, in accord with the

278 postulated role of PFC as a top-down controller, we found that the activation of these LRG

279 projections drives object exploration behavior in mice. Overall, our study shows that these top-

280 down prefrontal projections can dynamically control the network state and emergent circuit

281 function in the dHPC, thereby altering the signal-to-noise ratio for specific neural representations

282 and eliciting corresponding changes in behavior. This answers long-standing questions about the

283 mechanisms and nature of top-down control in the limbic system.

\section{Relationship to previous work}

286 Multiple lines of evidence in humans, non-human primates, and rodents have suggested that PFC 287 can exert top-down control over information processing in the HPC, particularly during 288 behaviors involving spatial and object exploration (Brincat and Miller, 2015; Eichenbaum, 2017; 289 Jin and Maren, 2015; Place et al., 2016; Preston and Eichenbaum, 2013; Shin and Jadhav, 2016;

290 Sigurdsson and Duvarci, 2016; Yu and Frank, 2015). Importantly, previous studies have shown 291 that lesion and pharmacological inactivation of PFC severely impairs spatial navigation and 292 object exploratory behaviors, and also disrupts neuronal encoding in the HPC (Churchwell et al., 293 2010; DeVito and Eichenbaum, 2010; Floresco et al., 1997; Guise and Shapiro, 2017; Kyd and 294 Bilkey, 2003; Wang and Cai, 2006; Yoon et al., 2008). Nevertheless basic aspects of this process 295 have remained elusive. Specifically, the pathways mediating prefrontal top-down control have 296 not been identified, and it was not known whether the PFC acts by transmitting specific 297 information to the HPC vs. by modulating the network state and emergent circuit function. 298 Addressing these questions is crucially important, because interactions between the HPC and 
PFC have been implicated in so many behaviors and disorders, and because the role of the PFC in top-down control is largely taken for granted despite the paucity of knowledge about specific mechanisms. Our study addresses this major gap by revealing a novel anatomical substrate mediating prefrontal top-down control, and showing exactly how it regulates behavior via actions on hippocampal neurons, microcircuits, network dynamics, and information processing.

Our anterograde tracing experiments showed that the PFC LRG projections are concentrated in the dorsal HPC, relative to the intermediate and ventral parts portions of HPC. By contrast, the NR, which is known to mediate indirect PFC-HPC communication, preferentially innervates the intermediate and ventral HPC (Hoover and Vertes, 2012). This suggests that conjunctive information transfer via the direct PFC LRG pathway and the indirect $\mathrm{PFC} \rightarrow \mathrm{NR} \rightarrow \mathrm{HPC}$ pathway would allow PFC to orchestrate activity along the entire extent of the hippocampal dorsoventral axis. Interestingly, the hippocampal dorsoventral axis is functionally segregated with the dorsal HPC being crucially involved in spatial processing and the ventral HPC regulating emotions, fear, and anxiety (Fanselow and Dong, 2010). Therefore, an alternate possibility is that the direct PFC $\rightarrow$ dHPC LRG projections and indirect PFC $\rightarrow$ NR $\rightarrow$ ventral HPC projections may mediate fundamentally distinct aspects of top-down control over cognitive vs. emotional behaviors, respectively. Similar to the functional segregation along the hippocampal dorsoventral axis, the PFC can also be subdivided dorsoventrally, into functionally specialized subregions, e.g., anterior cingulate (ACC), prelimbic (PL), and infralimbic (IL) cortices. We found PFC-dHPC LRG projections originating from PL. Notably, the ACC also sends direct projections to the dHPC (Rajasethupathy et al., 2015). However, by targeting excitatory neurons in the CA3 subregion of the HPC, these previously described ACC-CA3 excitatory projections primarily regulate the retrieval of fear memories. While the role of ACC-CA3 projections in spatial exploratory behaviors has not been investigated, it is plausible that the direct projections originating from ACC and PL regions transmit parallel streams of information from the PFC to alter hippocampal activity during distinct behaviors. Future work will be necessary to elucidate how glutamatergic vs. GABAergic top-down projections originating from different regions of the PFC, and targeting different portions of the HPC potentially interact and/or complement each other.

Our study shows that PFC-dHPC LRG projections target inhibitory interneurons, but not excitatory pyramidal neurons, within the CA1 subregion. This preferential targeting of inhibitory interneurons is similar to what has been observed in previous studies of cortical LRG inputs to the HPC. Specifically, the entorhinal cortex, which is the primary interface between the HPC and the neocortex, sends LRG projections which target local interneurons in the HPC (Basu et al., 2016; Melzer et al., 2012). However, in contrast to effect we observed, whereby PFC-dHPC 
335 projections primarily act to reduce hippocampal feedforward inhibition. This raises the 336 possibility that feedforward inhibition may be a convergent pathway on which many LRG inputs 337 act to regulate hippocampal information processing. Feedforward inhibition represents an 338 attractive target, as it crucially regulates input-output gain, neuronal plasticity, and information encoding in hippocampal pyramidal neurons (Grienberger et al., 2017; McKenzie, 2018).

VIP interneurons in the HPC are a specialized class which disinhibit other GABAergic interneurons, thereby tending to promote increases in microcircuit activity. Accordingly, disinhibition mediated by hippocampal VIP interneurons has been implicated in gain control,

343 memory, selective attention, and goal-directed behaviors (Cunha-Reis and Caulino-Rocha, 2020;

344 Turi et al., 2019). While we found that the CA1 interneurons which receive PFC-dHPC LRG

345 inputs are electrophysiologically heterogeneous but tend to express VIP, understanding whether

346 their axons target specific inhibitory loci within the CA1 microcircuit will help us to further understand the detailed nature of their actions. This is important because VIP+ interneurons in

348 the CA1 subregion constitute electrophysiologically and morphologically diverse subtypes (Acsády et al., 1996a; 1996b; Chamberland and Topolnik, 2012). Prior work has also shown that specialized subpopulations of hippocampal VIP+ GABAergic neurons send long-range projections which innervate different parts of the hippocampal formation, and are recruited during specific oscillatory states (Francavilla et al., 2018). It is possible that PFC-dHPC LRG projections target these long-range projecting VIP neurons, contributing to their state-dependent patterns of activity, and helping to produce some of the changes in network dynamics we observed here. Alternatively, the changes in hippocampal low-gamma oscillations we observed could be result from increases in feedforward inhibition.

\section{Relationship to disease}

359 Many neuropsychiatric disorders - including autism, schizophrenia, depression, and anxiety 360 disorders - are proposed to involve deficits in prefrontal functions and top-down control (Gilbert et al., 2008; Hare and Duman, 2020; Orellana and Slachevsky, 2013), and in particular, altered connectivity and communication between PFC and HPC (Cunniff et al., 2020; Godsil et al., 2013; Kupferschmidt and Gordon, 2018; Li et al., 2015; Sigurdsson et al., 2010). Abnormalities in GABAergic neuron structure and function have also been heavily implicated in the pathophysiology of neuropsychiatric disorders (Chattopadhyaya and Cristo, 2012; Marin, 2012; Paterno et al., 2020). PFC-dHPC LRG projections obviously represent a point of convergence

367 for these different mechanisms. Thus, abnormalities in PFC-dHPC LRG projections could 368 plausibly contribute to the disruptions in network oscillations, top-down control, and PFC-dHPC communication that occur in a variety of disease states. 
bioRxiv preprint doi: https://doi.org/10.1101/2021.03.01.433441; this version posted March 2, 2021. The copyright holder for this preprint (which was not certified by peer review) is the author/funder, who has granted bioRxiv a license to display the preprint in perpetuity. It is made available under aCC-BY-NC-ND 4.0 International license.

\section{Conclusion}

372 In summary, our study describes a novel anatomical pathway which plays a key role in direct

373 PFC-to-HPC communication. The unique features of these projections (i.e., long-range

374 GABAergic, disinhibitory interneuron targeting) enable PFC to dynamically alter emergent

375 network activity and information processing in the HPC, and thereby exert top-down control 376 over exploratory behavior. 


\section{Acknowledgements}

378 We thank M. Sharma and H. Seifikar for technical support. We are grateful to L. Frank, M.

379 Kheirbek, and members of the Sohal laboratory for comments on earlier versions of this

380 manuscript. This work was supported by National Institute of Mental Health (R01MH106507

381 and RO1MH117961 to V.S.S.) and 2018 NARSAD Young Investigator Grant from Brain \&

382 Behavior Research Foundation (Leichtung Family investigator, R.M.). Confocal images were

383 acquired at the Nikon Imaging Center at the University of California San Francisco.

\section{Author contributions}

386 R.M. and V.S.S. designed the experiments and analyses. R.M. performed all experiments and 387 analyzed the data, except that R.M. and Y.L. performed immunohistochemistry. S.S. generated 388 pilot histology data for anterograde tracing experiments. R.M. and V.S.S. wrote the manuscript.

\section{Competing interests}

391 The authors declare no competing interests. 


\section{MAIN FIGURES AND LEGENDS}

A AAV5-EF1a-DIO-eYFP

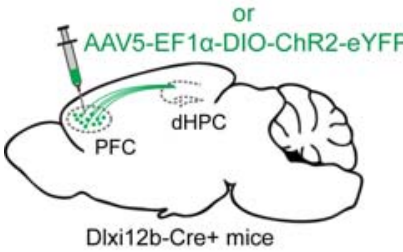

C

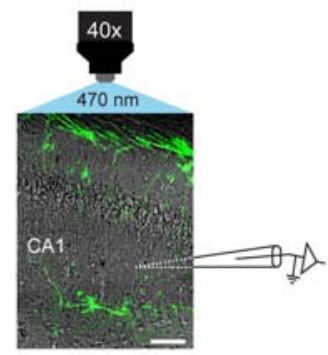

dHPC

$\mathbf{E}$

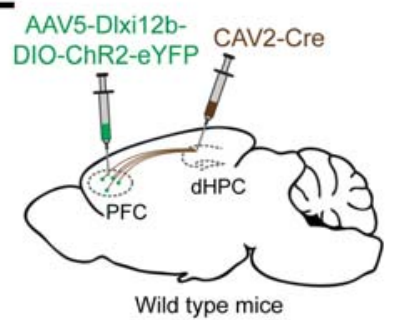

G
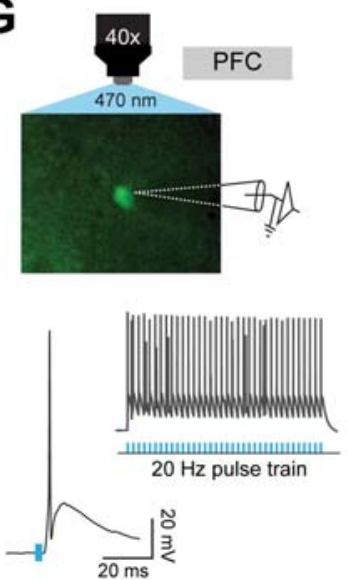

B

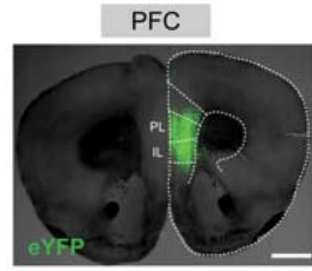

D
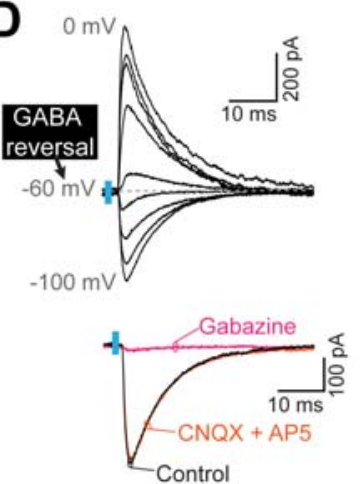

$\mathbf{F}$

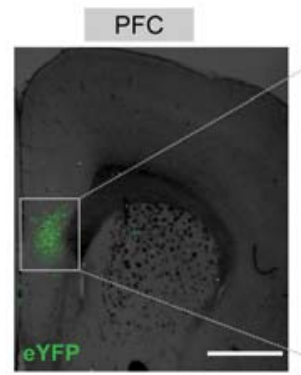

H

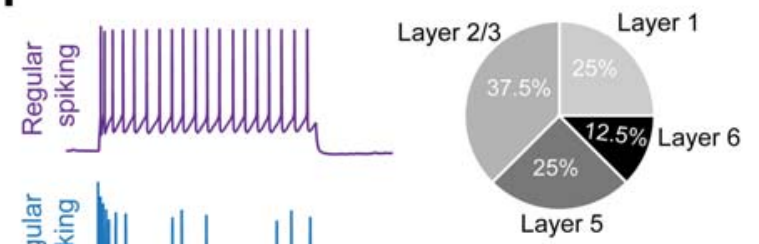

Regular spiking

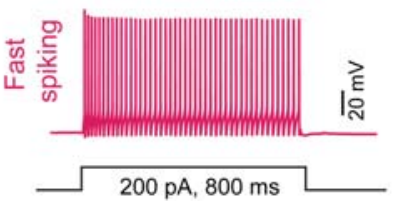

dHPC
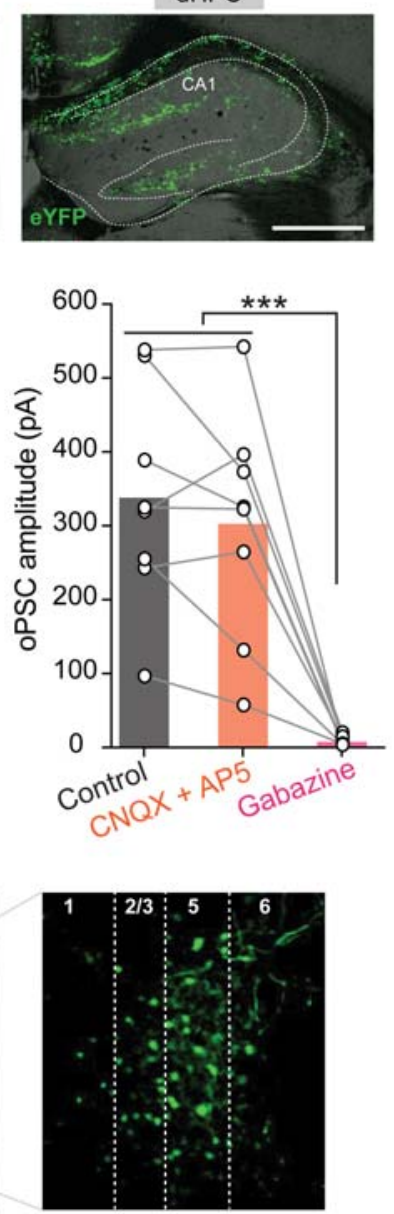

392 Figure 1: A heterogeneous population of PFC inhibitory neurons sends direct LRG 393 projections to the dHPC. 
394 (A) Schematic illustrating the anterograde tracing strategy. Cre-dependent eYFP or ChR2-eYFP virus was injected into the PFC of Dlxi12b-Cre+ mice.

396 (B) Representative images showing eYFP+ PFC GABAergic neurons (left) and eYFP+ axonal

397 fibers in the dHPC (right). Scale bars, $1 \mathrm{~mm}$ and $0.5 \mathrm{~mm}$, respectively. Prelimbic cortex (PL), 398 infralimbic cortex (IL), and hippocampal CA1 regions are labeled.

399 (C) Overlaid fluorescent and DIC images of a hippocampal section showing ChR2-eYFP+ 400 axonal fibers (green) in dorsal CA1. During ex-vivo patch clamp recordings from hippocampal 401 neurons, ChR2+ LRG axons were optogenetically activated by pulses of blue light (5 ms, 470 $402 \mathrm{~nm}$ ) delivered through the 40x objective. Scale bar, $100 \mu \mathrm{m}$.

403 (D) Top left: example traces showing that optogenetically evoked postsynaptic currents (oPSCs) 404 in recipient CA1 neurons reverse at the GABA reversal potential (gray dashed line). Blue bars 405 denote light pulses. Bottom left: example oPSCs recorded from a CA1 neuron in control aCSF 406 (black), after adding CNQX + AP5 (orange), and after adding Gabazine (magenta). Right: oPSC 407 amplitudes were significantly reduced by Gabazine. Open circles represent data from individual 408 neurons $(\mathrm{n}=8)$ and bars represent averages; one-way ANOVA followed by Tukey's multiple 409 comparison test, $* * * \mathrm{p}<0.001$.

410 (E) Schematic demonstrating the intersectional strategy to target dHPC-projecting PFC LRG 411 neurons. Retrogradely transducing canine adenovirus type-2 Cre (CAV2-Cre) was injected into 412 dHPC, and a Cre-dependent AAV that drives expression of ChR2-eYFP using a GABAergic 413 neuron-specific enhancer (Dlxi12b) was injected into PFC.

414 (F) Representative images showing ChR2-eYFP expression in dHPC projecting GABAergic 415 neurons in PFC. White dotted box in the left image corresponds to the magnified image shown in 416 right. Numbers indicate the cortical layers. Scale bar, $1 \mathrm{~mm}$.

417 (G) Top: representative image showing ex-vivo patch clamp recording obtained from ChR2418 eYFP expressing PFC-dHPC LRG neuron. Bottom: example traces showing PFC-dHPC LRG 419 neuron firing elicited in response to a single light pulse $(5 \mathrm{~ms}, 470 \mathrm{~nm})$ or a $20 \mathrm{~Hz}$ train.

420 (H) Left: example voltage responses of PFC-dHPC LRG neurons to depolarizing current 421 injections. Top right: pie chart showing the laminar distribution of recorded PFC-dHPC LRG 422 neurons. Bottom right: pie chart showing the percentage of PFC-dHPC LRG neurons with 423 various physiological properties.

424 See also Figures S1, S2 and Table S1. 


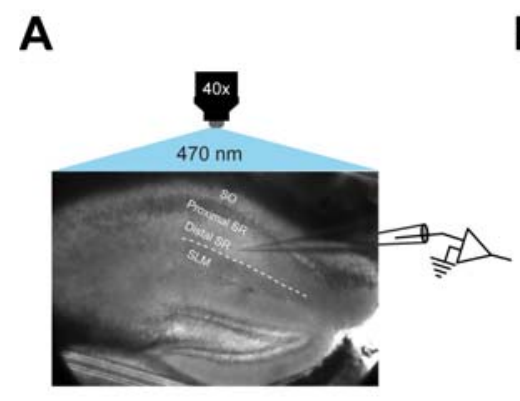

B
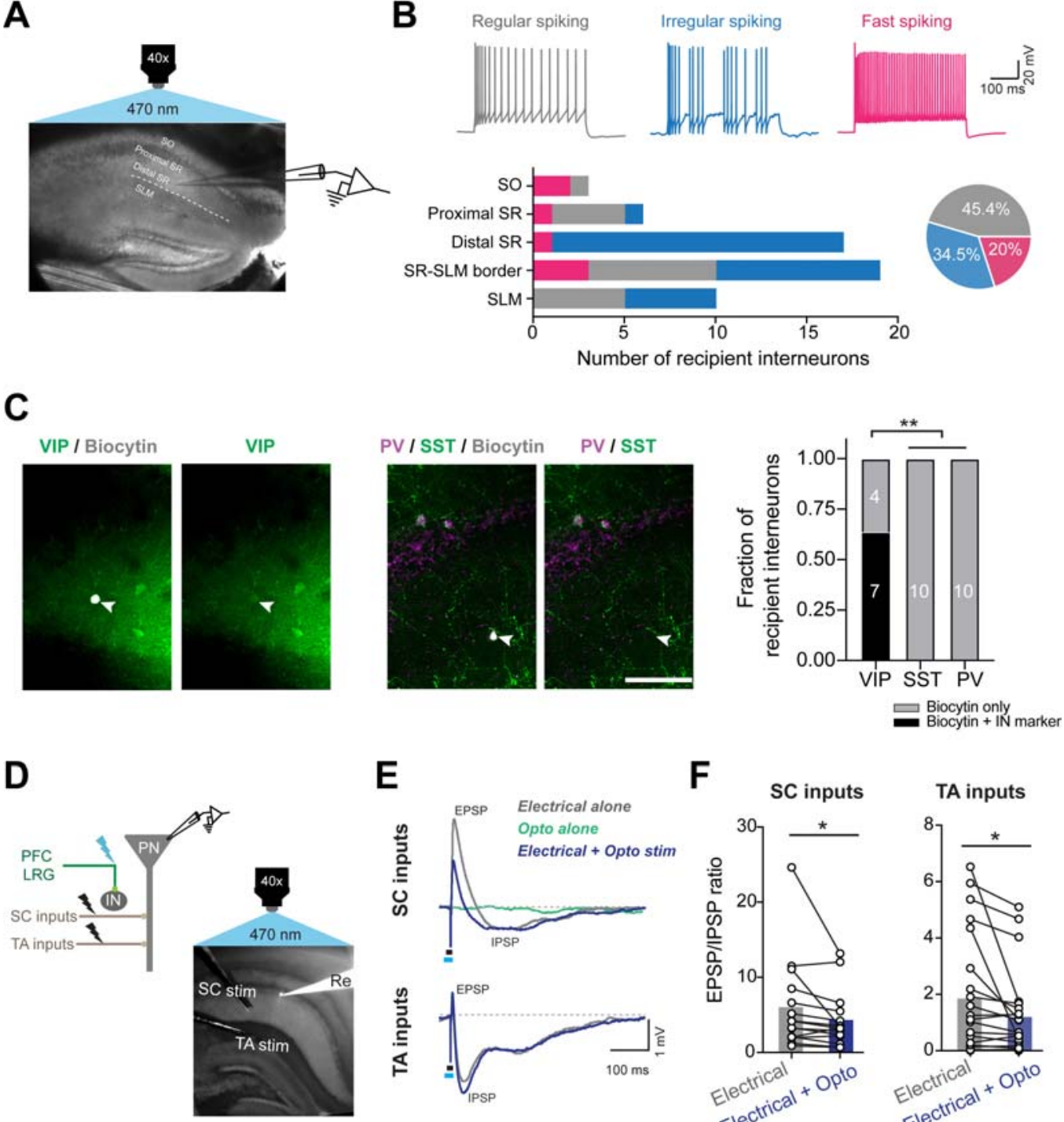

E

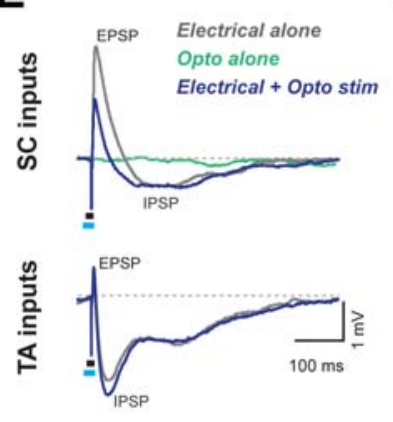

$\mathbf{F}$
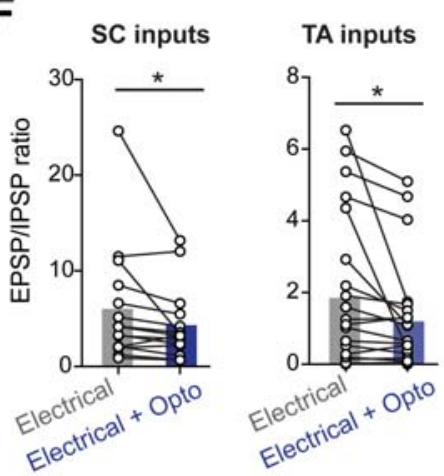

G
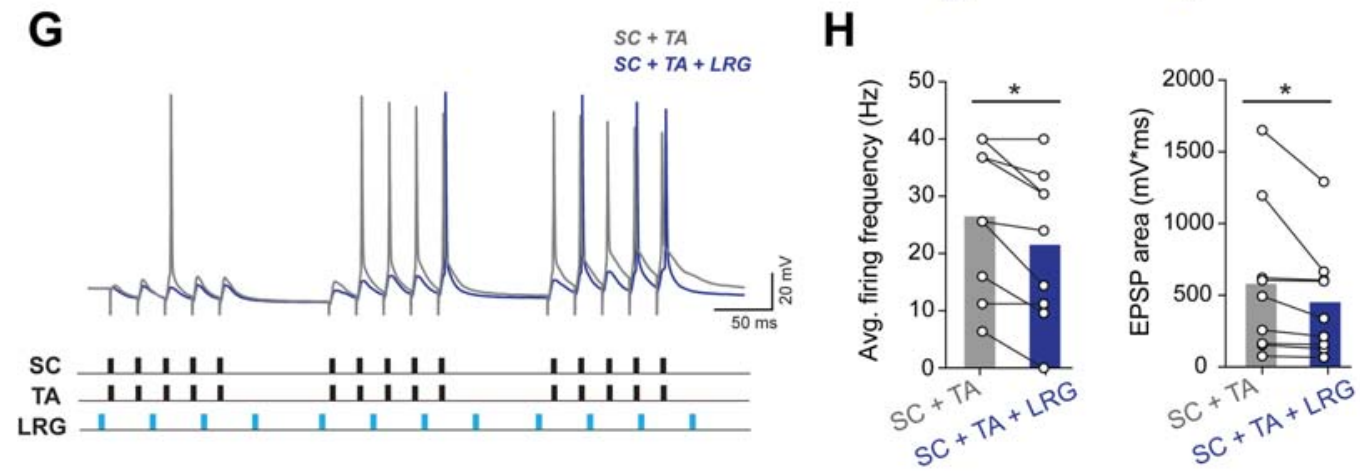

Figure 2: PFC LRG projections preferentially target interneuron-selective interneurons (ISIs) and increase feedforward inhibition in the CA1 microcircuit.

427 (A) Example DIC image of a hippocampal slice showing ex-vivo patch clamp recording from a 428 CA1 neuron during optogenetic stimulation of PFC LRG projections. Different layers of CA1 are 
labeled; stratum oriens (SO), stratum radiatum (SR), and stratum lacunosum-pyramidale (SLM); dashed white line represents the border between SR and SLM.

(B) Top: example voltage responses to depolarizing current injections for CA1 neurons which receive input from PFC LRG projections. Regular spiking (gray), irregular spiking (blue), and fast spiking properties (magenta) are observed among recipient CA1 neurons. Bottom left: number of recipient interneurons with fast spiking, regular spiking and irregular spiking physiology at different laminar locations in CA1 is plotted. Bottom right: pie chart showing the percentage of recipient CA1 neurons with regular spiking (25/55), irregular spiking (19/55), and fast spiking (11/55) properties.

438 (C) Left: Representative images showing staining for inhibitory neuron (IN) markers (VIP, PV or SST) in recipient CA1 neurons filled with biocytin. Scale bar, $100 \mu \mathrm{m}$. Right: fraction of recipient neurons which stained positive for VIP or (in separate sections) for PV or SST. More recipient neurons stained positive for VIP vs. PV or SST (Chi-square test, ** $\mathrm{p}<0.01$ ). (D) Top left: Schematic showing the experimental configuration. Right: example hippocampal image showing Alexa-594 filled recording electrode ( $\mathrm{Re})$ targeting a CA1 pyramidal neuron

444 (PN). Stimulating electrodes were placed in SR and SLM to stimulate Schaffer collateral (SC stim) or temporoammonic (TA stim) inputs, respectively. Brief pulses of blue light (5ms, 470 $\mathrm{nm}$ ) delivered through the 40x objective were used to optogenetically stimulate PFC LRG 447 projections.

448 (E) Excitatory and inhibitory postsynaptic potentials (EPSPs and IPSPs) elicited by electrical 449 stimulation of SC or TA inputs (black bar) in the presence or absence of optogenetic stimulation 450 of PFC-dHPC LRG projections (cyan bar). Gray traces show responses to electrical stimulation 451 alone, blue traces show responses during combined electrical + optogenetic stimulation, and 452 green trace shows response to optogenetic stimulation alone.

453 (F) Right: optogenetic stimulation of PFC-dHPC LRG projections significantly decreased EPSP 454 to IPSP ratios for both SC $(n=15$ cells $)$ and TA $(n=22$ cells) inputs. Lines connect values from 455 individual neurons and bars represent averages; two-way paired t-test, ** $\mathrm{p}<0.01,{ }^{*} \mathrm{p}<0.05$.

456 (G) Example voltage responses of CA1 PN to coincident theta-burst stimulation (TBS) of SC 457 and TA inputs (black bars) combined with $20 \mathrm{~Hz}$ optogenetic stimulation of PFC LRG 458 projections (cyan bars). Gray trace shows voltage response to SC and TA electrical stimulation, 459 and blue trace denotes response during SC and TA electrical stimulation + optogenetic 460 stimulation of PFC LRG projections.

461 (H) Average firing frequency (left) and EPSP area (right) during TBS of SC and TA inputs are 462 reduced by concomitant optogenetic stimulation of PFC LRG projections. Open circles represent 463 individual neurons $(\mathrm{n}=9)$ and bars represent averages; two-way paired t-test, $* \mathrm{p}<0.05$.

464 See also Figure S3 and Table S2. 
A

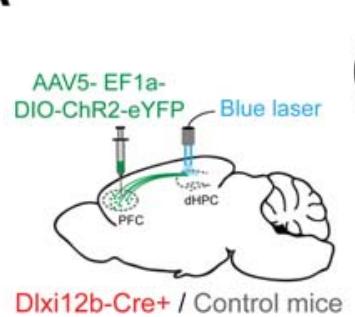

C

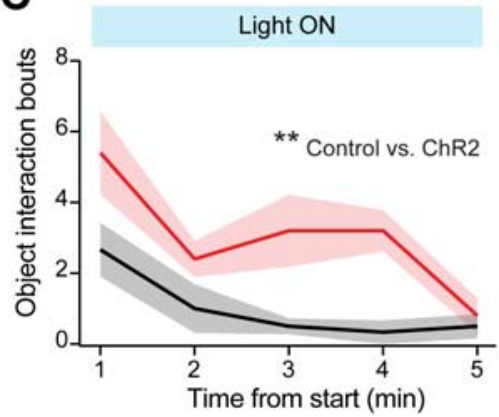

B

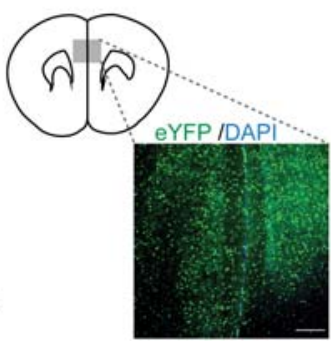

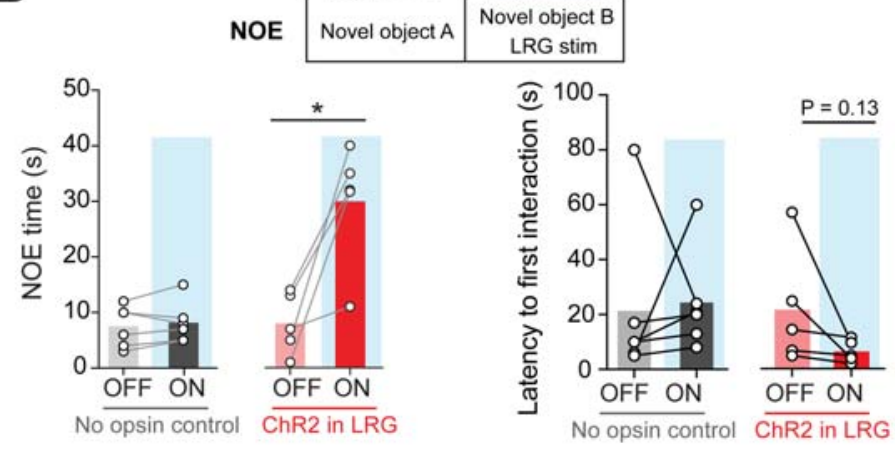

D

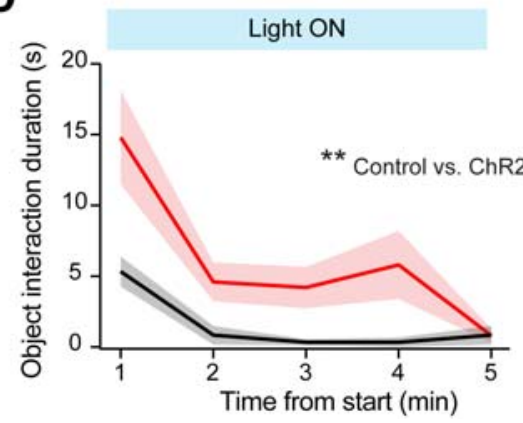

$\mathbf{E}$

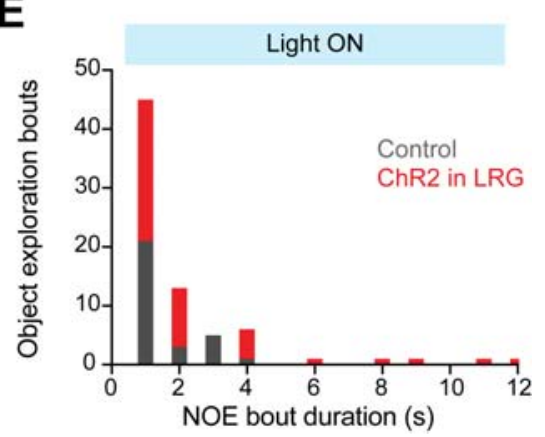

\section{Figure 3: Activating PFC-dHPC LRG projections increases novel object exploration.}

466 (A) Left: schematic illustrating the experimental design. Bilateral injections of Cre-dependent

467 ChR2-eYFP virus into the PFC of Dlxil2b-Cre+ mice or Cre-negative (control) mice. Bilateral

468 optical fibers were implanted over dHPC. Right: representative image showing ChR2-eYFP

469 expression in PFC GABAergic neurons. Scale bar, $200 \mu \mathrm{m}$.

470 (B) Top: novel object exploration (NOE) was measured in the presence (Light ON) or absence 471 (Light OFF) of optogenetic stimulation (473nm, 1.5-2 mW/fiber, $5 \mathrm{~ms}$ pulses at $20 \mathrm{~Hz}$ ). Bottom

472 left: optogenetic stimulation significantly increased NOE time in ChR2+ mice $(\mathrm{n}=5)$ but not in 473 control mice $(n=6)$. Bottom right: Latency to first interaction with a novel object for $\mathrm{ChR} 2+$ and 474 control mice is plotted. Open circles represent values from individual mice and bars indicate 475 averages. Two-way paired t-test, $* \mathrm{p}<0.05$.

476 (C-D) Number of object interaction bouts within 1-minute bins (C), and duration of object 477 interaction within 1-minute bins (D), over the duration of a 5-minute NOE testing session. Two478 way repeated measures ANOVA, $* * \mathrm{p}<0.01$.

479 (E) Distribution of NOE bout durations during optogenetic stimulation of PFC LRG projections 480 in control and ChR2-expressing mice.

481 See also Figure S4. 
A
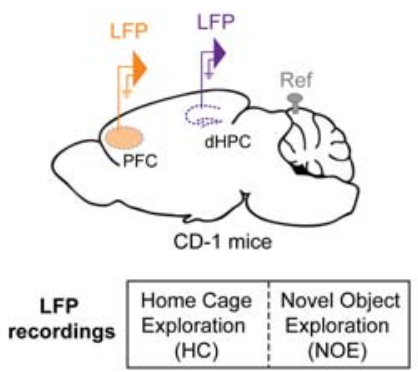

C
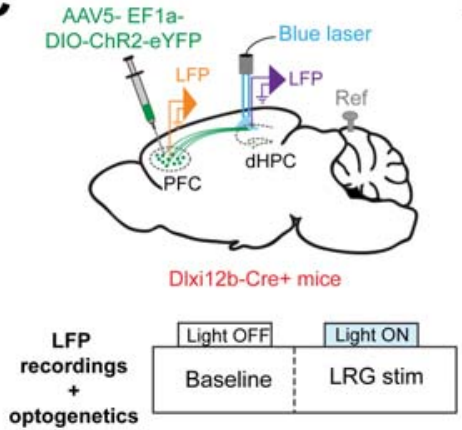

\section{B}
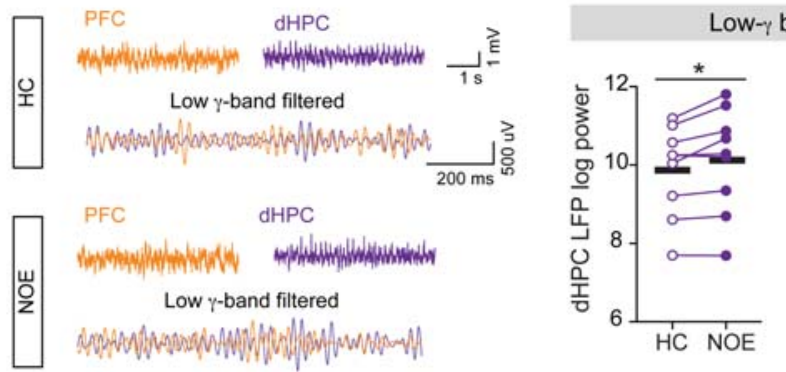

Low-y band network activity during NOE
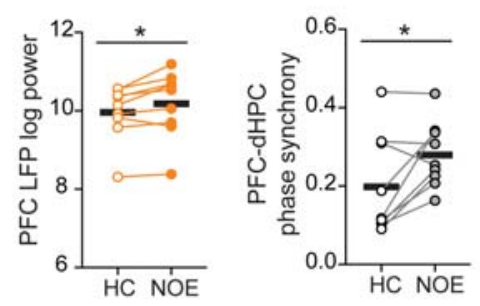

\section{D}

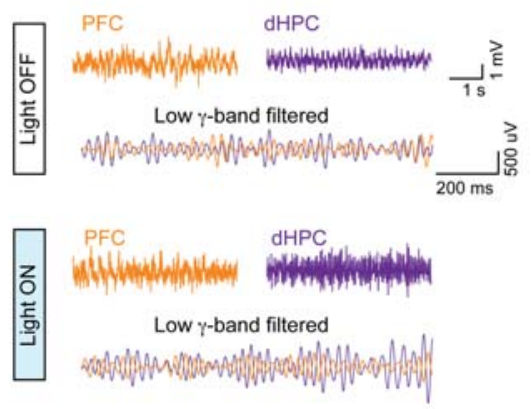

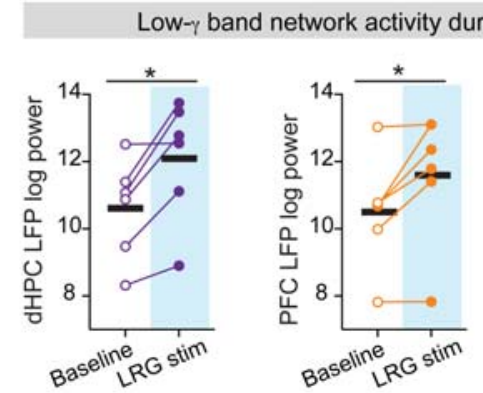

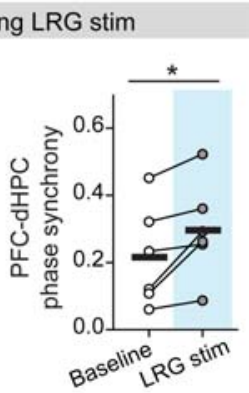

Figure 4: Activating PFC-dHPC LRG projections increases low-gamma oscillations

\section{associated with NOE.}

484 (A) Top: LFP electrodes were implanted in PFC and dHPC, reference electrode was implanted over the cerebellum. Bottom: LFPs recorded while mice were in their home cages (HC) were compared to LFPs recorded during NOE epochs.

(B) Left: example raw and low-gamma (low- $\gamma$ ) frequency filtered LFPs recorded during HC and NOE epochs are shown. Right: low- $\gamma$ power in dHPC and PFC and low- $\gamma$ phase synchrony between PFC and dHPC were all significantly higher during NOE. Open and filled circles represent data from individual mice $(n=9)$ and solid black lines represent averages. Two-way 491 paired t-test, $*$ p $<0.05$.

492 (C) Top: Schematic illustrating the experimental design for combined optogenetic stimulation and LFP recordings. Cre-dependent ChR2-eYFP virus was bilaterally injected into the PFC of

495 implanted in PFC and dHPC; reference electrode was implanted over the cerebellum. Bottom:

496 LFPs were recorded during baseline epochs (Light OFF) or LRG stimulation epoch (Light ON;

$497473 \mathrm{~nm}, 5 \mathrm{~ms}$ pulses at $20 \mathrm{~Hz}$ ).

498 (D) Left: example raw and low- $\gamma$ band filtered LFPs recorded during Light OFF and Light ON epochs. Right: low- $\gamma$ power in dHPC and PFC and low- $\gamma$ phase synchrony between PFC and dHPC were all significantly higher during LRG stim (Light ON) epoch. Open and filled circles represent data from individual mice $(n=6)$ and solid black lines represent averages. Two-way

502 paired t-test, $* \mathrm{p}<0.05$.

503 See also Figure S5. 


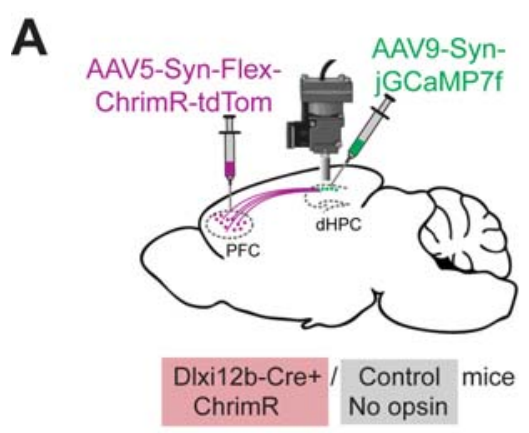

C

$\mathrm{Ca}^{2+}$ imaging during NOE testing
\begin{tabular}{|l|c|c|}
\hline Day 1 & HC & $\begin{array}{c}\text { NOE } \\
\text { Object A }\end{array}$ \\
\hline LRG stim OFF & HC & NOE Object B \\
Day 2 & HC & $\begin{array}{c}\text { HC stim } \\
\text { LRG stim }\end{array}$ \\
\hline LRG stim ON \\
\hline
\end{tabular}
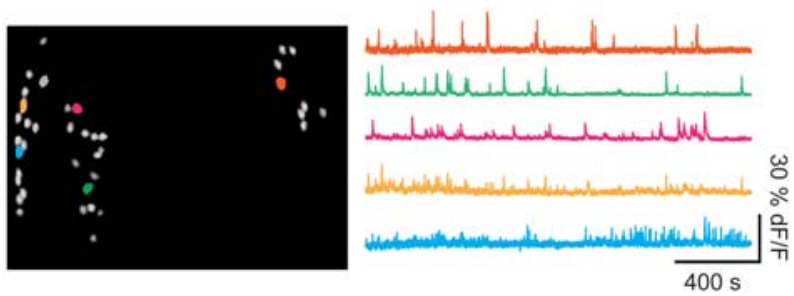

B
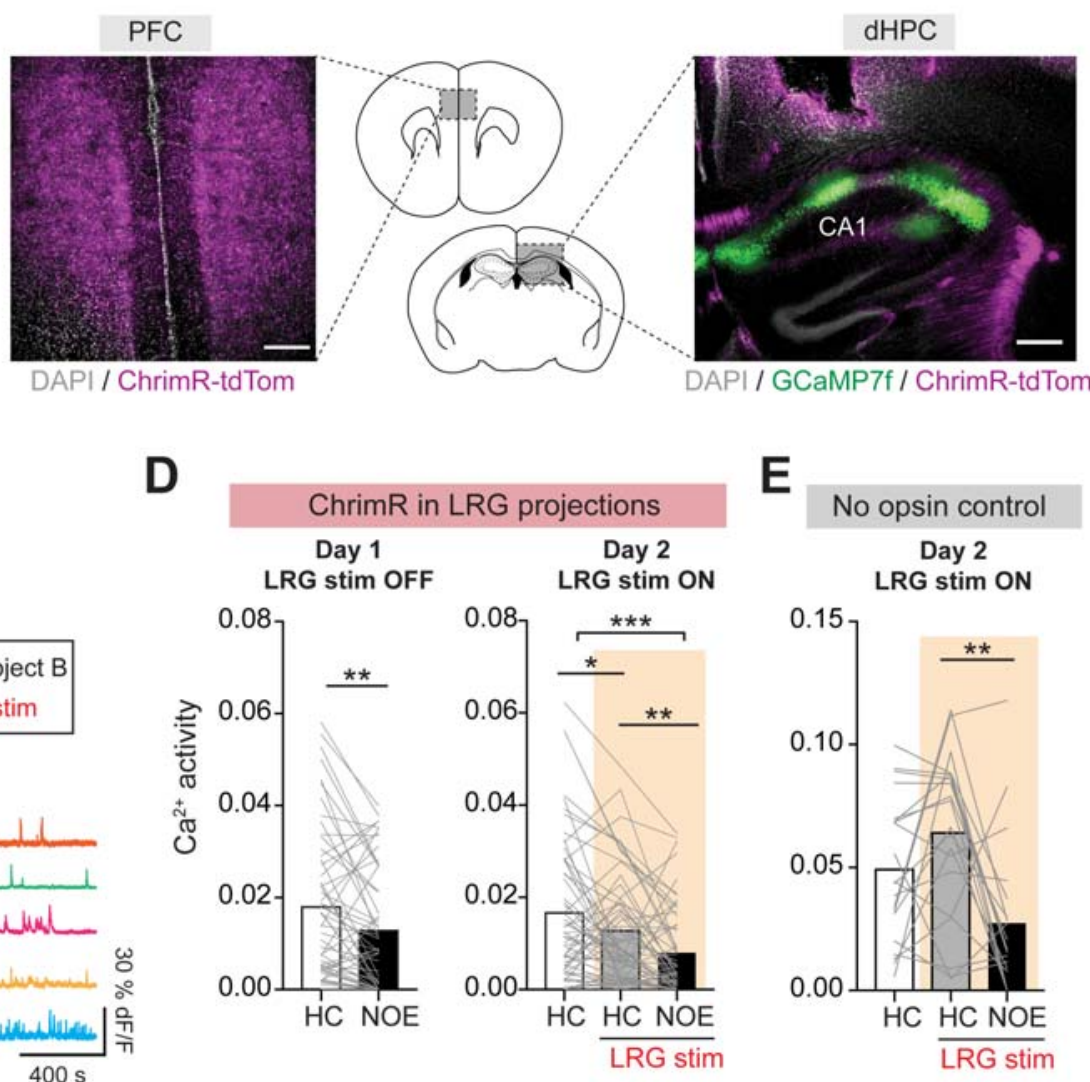

Figure 5: PFC-dHPC LRG projections shape CA1 neuronal activity during object exploration.

506 (A) Strategy for in vivo $\mathrm{Ca}^{2+}$ imaging and optogenetic stimulation. Cre-dependent ChrimsonRtdTomato (ChrimR-tdTom) virus was injected into the PFC of Dlxi12b-Cre+ and Cre-negative (control) mice; jGCamp7f virus was injected into dorsal $\mathrm{CA} 1$, and $\mathrm{Ca}^{2+}$ activity was imaged through an implanted GRIN lens connected to a miniscope.

(B) Left: DAPI stained coronal section showing ChrimR-tdTom expression in PFC GABAergic neurons. Right: DAPI stained dHPC section showing jGCaMP7f expression in CA1 neurons and

512 ChrimR-tdTom expression in PFC-dHPC LRG axonal fibers. Scale bars, $0.5 \mathrm{~mm}$.

(C) Top: on day 1, CA1 $\mathrm{Ca}^{2+}$ activity was measured during home cage (HC) and NOE epochs. On day 2, following an initial period of imaging in $\mathrm{HC}$ without optogenetic stimulation, $\mathrm{Ca}^{2+}$ imaging was combined with optogenetic stimulation of PFC-dHPC LRG projections (590-650 $\mathrm{nm}, \sim 2 \mathrm{~mW}, 5 \mathrm{~ms}$ pulses at $20 \mathrm{~Hz}$ ) during $\mathrm{HC}$ and NOE epochs. Bottom left: regions of interest (ROIs) corresponding to neurons from a representative $\mathrm{Ca}^{2+}$ imaging session. Bottom right: extracted $\mathrm{dF} / \mathrm{F} \mathrm{Ca}^{2+}$ transients from example dorsal CA1 neurons. Colors of the traces on the right correspond to the colored ROIs on the left.

(D) Left: $\mathrm{Ca}^{2+}$ activity in CA1 neurons was significantly reduced during NOE. Each gray line represents a single neuron and bars represent the mean ( $\mathrm{n}=55$ neurons from 2 mice); two-way 
522 paired t-test. Right: During the HC epoch on day 2, optogenetic stimulation of PFC-dHPC LRG 523 projections reduced $\mathrm{CA} 1 \mathrm{Ca}^{2+}$ activity. On Day 2, activity was then further reduced during NOE 524 ( $\mathrm{n}=59$ neurons, 2 mice). One-way ANOVA followed by Tukey's multiple comparison test; *** $525 \mathrm{p}<0.001, * * \mathrm{p}<0.01, * \mathrm{p}<0.05$.

526 (E) Same as $\mathbf{D}$ for control (opsin-negative) mice. Light delivery alone did not affect $\mathrm{Ca}^{2+}$ 527 activity. One-way ANOVA followed by Tukey's multiple comparison test; ** $\mathrm{p}<0.01$.

528 See also Figure S6. 
A

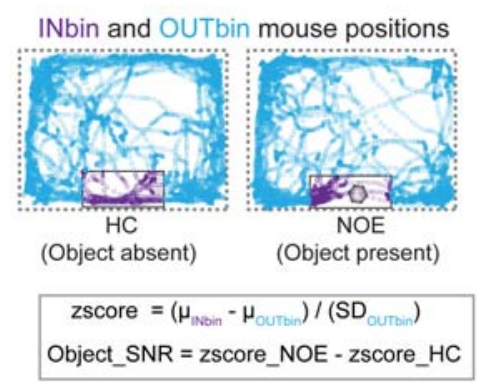

D
B

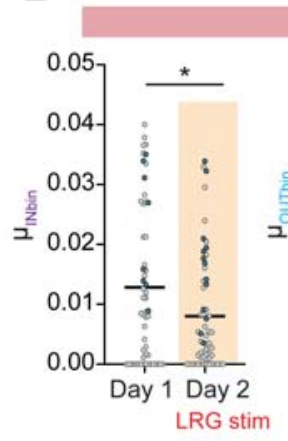

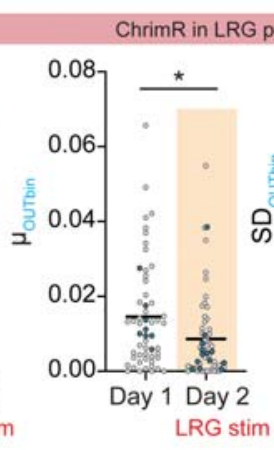
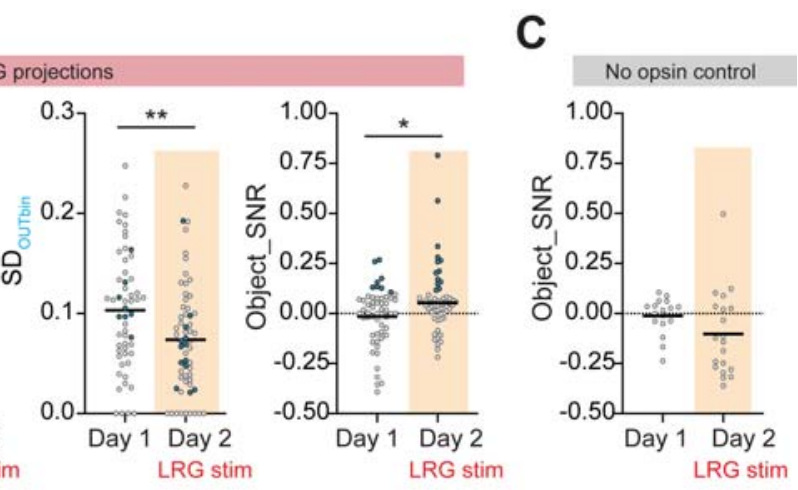

E

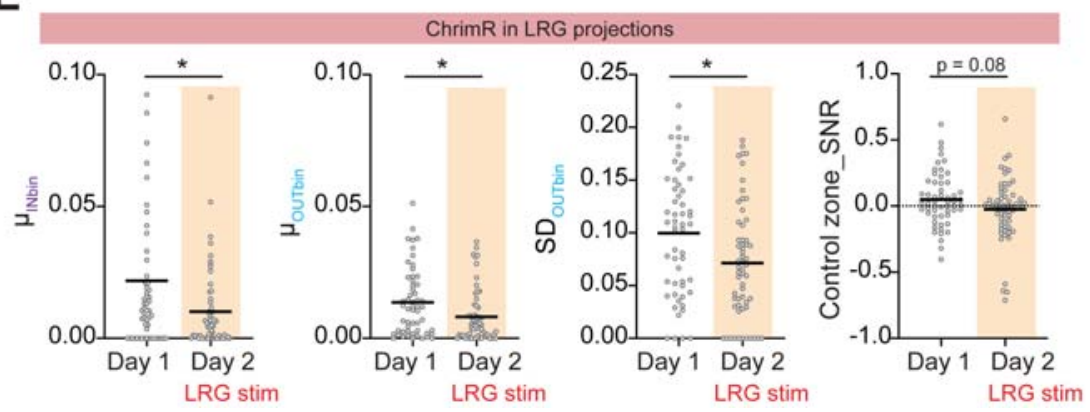

529 Figure 6: PFC-dHPC LRG projections increase neuronal signal-to-noise ratio for 530 representation of object location in CA1.

531 (A) Top: Frame-by-frame mouse positions during HC (left) and NOE (right) epochs are plotted.

532 Example data from day 2 recording session is shown. Blue circles denote frames where mouse was outside the object zone ('OUTbin'). Purple circles denote frames where mouse was in the object zone ('INbin'). Gray shaded hexagon indicates the location of the novel object during 535 NOE epoch and rectangle with black solid lines denotes the object zone. Bottom: z-scored $\mathrm{Ca}^{2+}$ 536 activity was calculated as the difference between the mean $\mathrm{Ca}^{2+}$ activity when mouse was within 537 ( $\left.\mu_{\text {INbin }}\right)$ vs. outside ( $\left.\mu_{\text {OUTbin }}\right)$ the object zone, divided by the standard deviation of activity outside 538 the object zone ( $\mathrm{SD}_{\text {OUTbin }}$ ). The difference in $\mathrm{z}$-scored $\mathrm{Ca}^{2+}$ activity between $\mathrm{HC}$ to NOE epochs 539 was used to compute Object_SNR.

540 (B) Optogenetic stimulation of PFC-dHPC LRG projections significantly reduced $\mu_{\mathrm{INbin}}$, $\mu_{\mathrm{OUTbin}}$, and SDouTbin. Object_SNR was increased on day 2 compared to day 1 in opsin-expressing mice.

542 Empty gray circles represent from individual neurons and horizontal black lines show means.

543 Filled blue circles indicate neurons exceeding an arbitrary threshold for Object_SNR (>0.1) to

544 illustrate $\mu_{\mathrm{INbin}}, \mu_{\mathrm{OUTbin}}$, and $\mathrm{SD}_{\mathrm{OUTbin}}$ for these high-SNR neurons. Two-way unpaired t-test; $* * \mathrm{p}$ $545<0.01, * \mathrm{p}<0.05$.

546 (C) Object_SNR in control (opsin negative) mice was not affected by light delivery.

547 (D) Frame-by-frame mouse positions during HC (left) and NOE (right) epochs are plotted. 548 Example data from day 2 recording session is shown. Blue circles denote frames where mouse 549 was away from a control zone ('OUTbin') that was the mirror image of the object zone, but on 
550 the opposite side of the cage. Purple circles denote frames where mouse was in the control zone 551 ('INbin'). Gray shaded hexagon denotes the novel object location in NOE epoch and rectangle 552 with black solid lines denotes the control zone.

553 (E) For the control zone, optogenetic stimulation of PFC-dHPC LRG projections significantly 554 reduced $\mu_{\mathrm{INbin}}, \mu_{\mathrm{OUTbin}}$, and $\mathrm{SD}_{\text {OUTbin }}$ (similar to the object zone). However, SNR computed for 555 the control zone was not affected by optogenetic stimulation of LRG projections (in contrast to 556 the object zone). Empty gray circles represent values from individual neurons and black lines 557 represent mean values. Two-way unpaired t-test; * $\mathrm{p}<0.05$. 


\section{METHODS}

\section{Animals}

560 All animal care procedures and experiments were conducted in accordance with the National Institutes of Health guidelines and approved by the Administrative Panels on Laboratory Animal

562 Care at the University of California, San Francisco. Mice were housed in a temperature563 controlled environment $\left(22-24{ }^{\circ} \mathrm{C}\right)$ with ad libitum access to food and water. Mice were reared

564 in normal lighting conditions (12-h light/dark cycle). Adult mice from the following lines were 565 used: Dlxi12b-Cre (Potter et al., 2009) and wild-type CD-1.

\section{Virus and retrograde tracer injections}

568 Mice were anesthetized with isoflurane and placed on a stereotaxic frame (David Kopf Instruments). An incision was made to expose the skull, and bregma and lambda were used as references to align the skull. Body temperature was maintained using a heating pad. Virus was injected (at the rate of $100 \mathrm{nl} / \mathrm{min}$ ) with a microinjection syringe (Nanofil $10 \mu \mathrm{l}$ with 35 gauge needle, World Precision Instruments) connected to a microsyringe pump (World Precision Instruments, UMP3 UltraMicroPump). Coordinates for injections into PFC were (in mm, relative to Bregma) 1.8 anterior-posterior (AP), \pm 0.3 mediolateral (ML), -2.4 dorsoventral (DV); and coordinates for injections into dHPC were -1.35 AP, $\pm 0.65 \mathrm{ML},-1.5 \mathrm{DV}$.

For anterograde tracing, ChR2 assisted circuit-mapping and optogenetic stimulation experiments, either AAV5-EF1 $\alpha$-DIO-eYFP virus or AAV5-EF1 $\alpha$-DIO-ChR2-eYFP virus (UNC

578 Vector core, $650 \mathrm{nl}$ ) was injected into PFC of Dlxi12b-Cre+ mice and Cre-negative mice. For intersectional labeling of dHPC projecting PFC LRG neurons, CAV2-Cre virus (del Rio et al., 2019; Hnasko et al., 2006) (650 nl) was injected in the dHPC and AAV5-Dlxi12b-BG-DIOChR2-eYFP virus (Lee et al., 2014) (650 nl) was injected in the PFC of CD-1 mice. For retrograde labeling of dHPC projecting PFC LRG neurons, Alexa Flour 594 Cholera toxin beta subunit conjugate (CTb-594, Invitrogen; 0.5\% w/v, 400-500 nl) was injected in dHPC of CD-1 mice. After virus or tracer injection, the microinjector needle was left in place for 5-6 min before being removed from the brain. Mice were sutured (if receiving viral/tracer injection only) and were allowed to recover on a heated pad until ambulatory.

\section{Optic fiber implantation}

589 Following bilateral AAV5-EF1 $\alpha$-DIO-ChR2-eYFP virus injection in PFC, dual fiber-optic 590 cannulas (Doric lenses; 200/240 mm, 0.22NA) were implanted in dHPC (-1.35 AP, \pm 0.65 ML, 591 1.4 DV). During these surgeries, the skull was scored with a scalpel to improve implant 592 adhesion. We waited at least 7 weeks after surgery to allow time for viral expression. 


\section{Optogenetic stimulation of PFC-dHPC LRG projections}

595 A $473 \mathrm{~nm}$ blue laser (OEM Laser Systems, Inc.) was coupled to the dual fiber-optic cannula 596 (implanted in dHPC) through a dual fiber-optic patch cord (Doric Lenses, Inc.), and was controlled via a function generator (Agilent 33500B Series Waveform Generator). Laser power

598 was adjusted such that the final light power was 3-4 $\mathrm{mW}$ total, summed across both fibers, and averaged over $20 \mathrm{~Hz}$ light pulses (5 ms duration).

\section{Behavioral assays}

602 After sufficient time for surgical recovery and viral expression, mice were handled and

603 habituated for multiple days (3-5 days). Briefly, mice were first habituated to the behavioral

604 testing room for $30 \mathrm{~min}$ prior to handling each day. For 2-3 days before starting testing, mice

605 were habituated to the cable tethers in their home cage for $15 \mathrm{~min}$. The experimenter was blinded

606 to experimental groups during behavioral testing and scoring. A USB webcam (Logitech)

607 connected to a computer running ANY-maze (Stoelting Co.) was used to record behavior 608 movies. The position of mice was tracked using the built-in tracking in ANY-maze software. In

609 some experiments, mouse positions were tracked using trained neural networks in DeepLabCut

610 open-source software package (Mathis et al., 2018).

Novel object exploration

613 For measuring novel object exploration, one previously unexplored object was placed in the

614 home cage of an experimental mouse for $5 \mathrm{~min}$. A blinded observer manually scored the

615 following parameters: exploration time, bouts of exploration, and latency to the first exploration.

616 Objects used in our study were usually lego toys, dice, small plumbing connectors, and falcon

617 tube caps. For experimental mice with dual-fiber optic implants, two object interaction tests were

618 performed over two days: day 1 testing was performed without light stimulation, and day 2

619 testing was done during optogenetic stimulation of PFC-dHPC LRG projections. Cre-negative

620 mice (no opsin control) with dual-fiber optic implants underwent similar behavior testing 621 procedures.

\section{Social interaction test}

624 For social interaction test, a novel juvenile (3-4 week old) mouse of the same sex was introduced

625 in the home cage of an experimental mouse for $5 \mathrm{~min}$. A blinded observer manually scored the

626 time (in seconds) the experimental mouse spent with its nose in direct contact with the novel 627 juvenile intruder. For all experimental mice, two social interaction tests were performed over two 628 days: day 1 testing was performed without light stimulation, and day 2 testing was done during 
optogenetic stimulation of PFC LRG projections.

Open field exploration test

632 Mice were placed in the center of a 50 x $50 \mathrm{~cm}$ open-field arena and were allowed to freely 633 explore for $12 \mathrm{~min}$. The testing time was divided into four (3 min) epochs. PFC-dHPC LRG 634 projections were optogenetically stimulated during the $2^{\text {nd }}$ and $4^{\text {th }}$ epochs. Distance traveled 635 during no stimulation (light OFF) and during optogenetic stimulation (light ON) epochs was 636 quantified using the ANY-maze tracking software. over 3 days. An apparatus with two identical chambers was used for RTPP testing. On day 1, mice were habituated to the apparatus for $15 \mathrm{~min}$. On day 2, mice were placed into one randomly

642 chosen chamber and the time spent in the two chambers was recorded. On day 3, one of the 643 chambers was randomly assigned as the stimulated chamber. When mice entered this chamber,

644 they received $20 \mathrm{~Hz}$ laser pulses $(473 \mathrm{~nm}, 3-4 \mathrm{~mW}, 5 \mathrm{~ms})$. The ratio of the time spent in the 645 simulated chamber vs. the non-stimulated chamber was used as the preference index. The sides 646 of the stimulated chambers were counterbalanced across all mice.

\section{LFP recordings: surgery and analysis}

649 Surgery

650 Mice were anesthetized with isoflurane and placed on a stereotactic frame. After cleaning, the 651 skull was scored with a scalpel to improve implant adhesion. For LFP recordings from wild-type 652 CD-1 mice, tungsten electrodes (Microprobes) were inserted into the PFC (1.8 AP, -0.3 ML, -2.4 $653 \mathrm{DV})$ and dHPC (-1.35 AP, -0.65 ML, -1.5 DV). For multisite LFP recordings combined with 654 optogenetics, one LFP electrode was implanted after AAV5-EF1 $\alpha$-DIO-ChR2-eYFP virus 655 injection into PFC of Dlxi12b-Cre+ mice. A custom-made optrode (optical fiber + electrode) 656 was implanted in dHPC to stimulate ChR2+ PFC-dHPC LRG axon terminals during LFP 657 recordings. To fabricate optrodes, a tungsten LFP recording electrode was affixed to one of the 658 fibers of the dual-fiber optic cannula such that the tip of the electrode protruded 200-300 $\mu \mathrm{m}$ 659 beyond the end the optic fiber (Lee et al., 2019). Reference and ground screws were implanted 660 above the cerebellum. Electrodes and screws were cemented to the skull with Metabond (Parkell) 661 and connected to a headstage for multi-channel recordings (Pinnacle). Following surgery, mice 662 were monitored postoperatively, given analgesics, and individually housed. 
665 LFP data were acquired at $2 \mathrm{KHz}$ and band-pass filtered from $0.5-150 \mathrm{~Hz}$. Electrode placements

666 were histologically confirmed. Analysis of LFP data was done using custom MATLAB

667 (Mathworks) scripts. Briefly, signals were imported into MATLAB and LFP log power (for both

668 channels) was calculated using the power spectral density output from the spectrogram function.

669 For phase-synchrony and amplitude covariance analysis, LFPs were FIR-filtered for different

670 frequency bands, then Hilbert transformed to yield the instantaneous amplitudes and phases. The

671 following frequency bands were compared: theta band (4-12 Hz), beta band (15-25 Hz), low-

672 gamma band $(25-55 \mathrm{~Hz})$, and high-gamma band $(65-85 \mathrm{~Hz})$.

673 To detect nonzero phase interdependencies (phase synchrony) between LFP signals

674 recorded at PFC and dHPC electrodes, we estimated the weighted Phase Lag Index (wPLI)

675 (Vinck et al., 2011) using the imaginary component of the cross-spectrum $\left(\mathrm{S}_{\mathrm{xy}}\right)$ (Equations 1.1

676 and 1.2). $\mathrm{A}_{\mathrm{x}}$ and $\mathrm{A}_{\mathrm{y}}$ are instantaneous amplitudes; and $\Phi \mathrm{x}$ and $\Phi y$ are instantaneous phases for

677 PFC and dHPC signals, respectively.

$$
S_{x y}=A_{x} A_{y} e^{i(\phi x-\phi y)}
$$

$$
w P L I=\frac{\left|\sum\right| \operatorname{imag}\left(s_{x y}\right)\left|\operatorname{sgn}\left(s_{x y}\right)\right|}{\sum\left|\operatorname{imag}\left(S_{x y}\right)\right|}
$$

The amplitude covariation between PFC and dHPC was calculated as the maximum normalized cross-correlation (xcorr function in MATLAB) of the instantaneous band-pass filtered amplitudes of LFP signals at each electrode. Coherence between LFP signals was computed using the mscohere function in MATLAB. Log power, wPLI, amplitude covariation, and coherence were calculated over short time intervals (at least $3 \mathrm{sec}$ in duration), i.e., the intervals during which a mouse was actively exploring an object or matched intervals during baseline periods when the mouse was in its home cage.

\section{Ex-vivo slice physiology}

691 Slice preparation

692 Adult mice were anesthetized with an intraperitoneal injection of euthasol and transcardially 693 perfused with an ice-cold cutting solution containing (in $\mathrm{mM}$ ) 210 sucrose, $2.5 \mathrm{KCl}, 1.25$ $694 \mathrm{NaH}_{2} \mathrm{PO}_{4}, 25 \mathrm{NaHCO}_{3}, 0.5 \mathrm{CaCl}_{2}, 7 \mathrm{MgCl}_{2}, 7$ dextrose (bubbled with $95 \% \mathrm{O}_{2}-5 \% \mathrm{CO}_{2}, \mathrm{pH}$ 695 7.4). Mice were decapitated and the brains were removed. For acute prefrontal sections: two 696 parallel cuts were made along the coronal plane at the rostral and caudal ends of the brains; 697 brains were mounted on the flat surface created at the caudal end; three coronal slices $(250 \square \mu \mathrm{m}$ 698 thick) were obtained using a vibrating blade microtome (VT1200S, Leica Microsystems Inc.). 699 Dorsal hippocampal (dHPC) slices were obtained using a blocking technique described 
700 previously (Malik et al., 2015). Briefly, dHPC slices were obtained by making a blocking cut at a

$70145^{\circ}$ angle from the coronal plane starting at the posterior end of the forebrain. A second blocking 702 cut was made at $45^{\circ}$ relative to the coronal plane, but starting from approximately one-third of 703 the total length of the forebrain (from the most anterior point). Brains were mounted on the flat 704 surface created by the first blocking cut. Approximately, 3 dorsal slices were obtained from each 705 hemisphere.

Slices were allowed to recover at $34 \square{ }^{\circ} \mathrm{C}$ for $30 \square \min$ followed by $30 \square$ min recovery at room temperature in a holding solution containing (in $\mathrm{mM}$ ) $125 \mathrm{NaCl}, 2.5 \mathrm{KCl}, 1.25 \mathrm{NaH}_{2} \mathrm{PO}_{4}$, $25 \mathrm{NaHCO}_{3}, 2 \mathrm{CaCl}_{2}, 2 \mathrm{MgCl}_{2}, 12.5$ dextrose, 1.3 ascorbic acid, 3 sodium pyruvate.

Ex-vivo patch clamp recordings

711 Somatic whole-cell current-clamp and voltage-clamp recordings were obtained as previously 712 described (Malik and Johnston, 2017; Malik et al., 2019). Briefly, submerged slices were 713 perfused in heated $\left(32-34 \square^{\circ} \mathrm{C}\right.$ ) artificial cerebrospinal fluid (aCSF) containing (in $\mathrm{mM}$ ): 125 $714 \mathrm{NaCl}, 3 \mathrm{KCl}, 1.25 \mathrm{NaH}_{2} \mathrm{PO}_{4}, 25 \mathrm{NaHCO}_{3}, 2 \mathrm{CaCl}_{2}, 1 \mathrm{MgCl}_{2}, 12.5$ dextrose (bubbled with $95 \%$ $715 \mathrm{O}_{2} / 5 \% \mathrm{CO}_{2}, \mathrm{pH} \sim 7.4$ ). Neurons were visualized using DIC optics (and eYFP fluorescence in a 716 few experiments) fitted with a 40x water-immersion objective (BX51WI, Olympus microscope). 717 During recordings from prefrontal slices, dHPC projecting PFC LRG neurons in all cortical 718 layers were identified by eYFP expression. During recordings from hippocampal slices, CA1 719 pyramidal neurons (PNs) and CA1 local inhibitory neurons (INs) were identified using laminar location (under DIC optics) and intrinsic properties of the recorded neurons.

Patch electrodes (2-3 $\square \mathrm{M} \Omega$ ) were pulled from borosilicate capillary glass of external 722 diameter $1 \square \mathrm{mm}$ (Sutter Instruments) using a Flaming/Brown micropipette puller (model P-2000, 723 Sutter Instruments). For current-clamp recordings, electrodes were filled with an internal 724 solution containing the following (in $\mathrm{mM}$ ): $134 \mathrm{~K}$-gluconate, $6 \mathrm{KCl}, 10 \mathrm{HEPES}, 4 \mathrm{NaCl}, 7 \mathrm{~K}_{2}$ 725 phosphocreatine, $0.3 \mathrm{Na}-\mathrm{GTP}$, and $4 \mathrm{Mg}-\mathrm{ATP}$ ( $\mathrm{pH} \sim 7.3$ adjusted with $\mathrm{KOH}$ ). Biocytin (Vector 726 Laboratories) was included $(0.1-0.2 \%)$ for subsequent histological processing of recipient CA1 727 neurons. For voltage-clamp recordings, the internal solution contained the following (in $\mathrm{mM}$ ): 728130 Cs-methanesulfonate, $10 \mathrm{CsCl}, 10 \mathrm{HEPES}, 4 \mathrm{NaCl}, 7$ phosphocreatine, $0.3 \mathrm{Na}-\mathrm{GTP}, 4 \mathrm{Mg}-$ 729 ATP, and 2 QX314-Br ( $\mathrm{pH} \sim 7.3$ adjusted with $\mathrm{CsOH}$ ). In a few recordings, $15 \mu \mathrm{m}$ AlexaFluor730594 (Invitrogen) was also added to the internal solution. Electrophysiology data were recorded 731 using Multiclamp 700B amplifier (Molecular Devices). Voltages have not been corrected for 732 measured liquid junction potential $(\sim 8 \square \mathrm{mV})$. Data collection was started 5-8 min after 733 successful transition to the whole-cell configuration. Series resistance and pipette capacitance 734 were appropriately compensated before each recording. Series resistance was usually 10$73520 \square \mathrm{M} \Omega$, and experiments were terminated if series resistances exceeded $25 \square \mathrm{M} \Omega$. 
Data analysis

738 Ex-vivo electrophysiology data were analyzed using custom routines written in IGOR Pro (Wavemetrics). Resting membrane potential (RMP) was measured in current-clamp mode immediately after reaching whole-cell configuration. Input resistance (Rin) was calculated as the slope of the linear fit of the voltage-current plot generated from a family of hyperpolarizing and depolarizing current injections ( -50 to $+20 \mathrm{pA}$, steps of $10 \square \mathrm{pA})$. Firing output was calculated as the number of action potentials (APs) fired in response to $800 \square \mathrm{ms}$ long depolarizing current injections (25-500 $\square \mathrm{pA}$ ). Firing frequency was calculated as the number of APs fired per second. Firing traces in response to $50 \mathrm{pA}$ current above the rheobase were used for analysis of single AP properties - AP threshold, maximum $d V / d t$ (rate of rise of AP), AP amplitude, AP half-width, and fast afterhyperpolarization (fAHP) amplitude. AP threshold was defined as the voltage at which the value of third derivative of voltage with time is maximum. Action potential amplitude was measured from threshold to peak, and the half-width was measured at half this distance. Fast afterhyperpolarization was measured from the threshold to the negative voltage peak after the AP. Index of spike-frequency accommodation (SFA) was calculated as the ratio of the last interspike interval to the first inter-spike interval.

Recorded inhibitory neurons (INs) in PFC and dorsal CA1 were classified as fast spiking, regular spiking or irregular spiking based on electrophysiological properties. Specifically, INs were classified as fast spiking if they met 3 out of the 4 following criteria: AP half-width was < $0.5 \square \mathrm{ms}$, firing frequency $>50 \square \mathrm{Hz}$, fAHP amplitude $>14 \square \mathrm{mV}$, and SFA index $<2$. Irregular spiking INs were initially visually identified based on their high variability in inter-spike interval and burst-like intermittent spiking properties. This classification was confirmed using a firing frequency threshold $(<50 \mathrm{~Hz})$ and/or a SFA index threshold (>2). Dorsal CA1 neurons were classified as pyramidal neurons if they satisfied the following criteria: cell body located in stratum pyramidale, AP half-width $>1 \mathrm{~ms}$, fAHP amplitude $<5 \mathrm{mV}$, and maximum firing frequency $<20 \mathrm{~Hz}$.

To measure optogenetically evoked spiking in ChR2-eYFP+ PFC INs and to measure optogenetically evoked postsynaptic currents (oPSCs) in CA1 neurons, ChR2 was stimulated using 5 $\square \mathrm{ms}$ long light pulses (maximum light power, $4 \square \mathrm{mW} / \mathrm{mm}^{2}$ ) generated by a Lambda DG4 high-speed optical switch with a 300 $\square$ W Xenon lamp (Sutter Instruments) and an excitation filter centered around $470 \square \mathrm{nm}$. Light pulses were delivered to the slice through a 40x objective (Olympus). To measure the reversal potential of oPSCs, the holding potentials were systematically varied from -100 to $+20 \mathrm{mV}$ in $10 \mathrm{mV}$ steps. The drugs applied were 6-cyano-7nitroquinoxaline-2,3-dione disodium salt hydrate (CNQX), 2-(3-carboxypropyl)-3-amino-6-(4 
772 (D-AP5) (Tocris). Drugs were prepared as concentrated stock solutions and were diluted in

773 ACSF on the day of the experiment.

774 To measure afferent input mediated feedforward excitation and inhibition in CA1 PNs, 775 bipolar stimulating electrodes (Microprobes) were placed at stratum radiatum (SR) and stratum 776 lacunosum-moleculare (SLM) to stimulate Schaffer collateral (SC) and temporo ammonic (TA) 777 inputs, respectively. The protocol for theta-burst stimulation (TBS) consisted of bursts with five 778 electrical stimulations $(40 \mathrm{~Hz})$ repeated at $5 \mathrm{~Hz}$. To measure the effect of PFC LRG inputs on 779 firing output and EPSP summation during TBS protocol, train of $470 \mathrm{~nm}$ light pulses $(20 \mathrm{~Hz}, 5$ $780 \mathrm{~ms}$ ) was delivered through the 40x objective. Firing frequency during TBS was calculated as the 781 average number or APs fired per burst, and summation was estimated as the area of the last 782 EPSP in the TBS train.

\section{In vivo $\mathrm{Ca}^{2+}$ imaging}

785 Surgery

786 Mice underwent two stereotactic surgeries. Cre-dependent AAV5-Syn-FLEX-ChrimsonR787 tdTomato virus (Addgene) was injected in PFC (1.8 AP, $\pm 0.3 \mathrm{ML},-2.4 \mathrm{DV})$ of Dlxil2b-Cre+ 788 and Cre-negative mice. Following this, 500-550 nl of AAV9-Syn-jGCaMP7f-WPRE virus 789 (diluted 1:2; Addgene) was injected in dorsal CA1 to express synapsin-driven calcium sensor jGCaMP7f (injection coordinates: -1.4 AP, +0.8 ML, -1.5 DV). After 3-4 weeks of viral expression, cortex overlying dorsal CA1 was slowly aspirated and a $1 \mathrm{~mm}$ diameter $\mathrm{x} 4 \mathrm{~mm}$ long integrated GRIN lens (Inscopix) was slowly advanced above the dorsal CA1 and cemented in place with Metabond dental cement. Mice were allowed to recover for at least 3 weeks before starting behavior and imaging experiments.

Combined $\mathrm{Ca}^{2+}$ imaging and optogenetics

797 Imaging data were collected using a miniaturized one-photon microscope (nVoke2; Inscopix Inc.). GCaMP7f signals ( $\mathrm{Ca}^{2+}$ activity) were detected using 435-460 nm excitation LED (0.1-0.2 $\mathrm{mW}$ ), and optogenetic stimulation of ChrimR expressing axons was performed using a second excitation LED centered around 590-650 nm (5 ms pulses at $20 \mathrm{~Hz}, 1-2 \mathrm{~mW}$ light power). $\mathrm{Ca}^{2+}$ movies were acquired at 20 frames per second, spatially downsampled (4x), and were stored for offline data processing.

Mice were placed into a large housing cage $(48 \times 35 \mathrm{~cm})$ for 2-3 days for $20 \mathrm{~min}$ where

804 they habituated to the scope. After habituation, mice underwent a two-day behavioral testing 805 protocol for recording NOE related $\mathrm{Ca}^{2+}$ activity in CA1 neurons. On day 1, mice were allowed 806 to explore the large home cage for $15 \mathrm{~min}$ (HC epoch). Following this, mice were allowed to 807 explore a novel object introduced in the cage for $15 \mathrm{~min}$ (NOE epoch). On day 2, mice were 
808 allowed to explore the home cage for $15 \mathrm{~min}$ (HC epoch) followed by optogenetic stimulation

809 during home cage exploration for $10 \mathrm{~min}$ (HC + LRG stim epoch). Mice were then allowed to

810 explore a novel object combined with optogenetic stimulation of PFC-dHPC LRG projections

811 (NOE + LRG stim epoch). The behavior of mice during different epochs was recorded using

812 ANY-maze software, and input TTL pulses from ANY-maze to nVoke2 acquisition software

813 were used to synchronize $\mathrm{Ca}^{2+}$ imaging and mouse behavior movies.

815 Data analysis

$816 \mathrm{Ca}^{2+}$ imaging movies were preprocessed using Inscopix Data Processing Software (IDPS;

817 Inscopix, Inc.). The video frames were spatially filtered (band-pass) with cut-offs set to 0.005

818 pixel $^{-1}$ (low) and 0.5 pixel $^{-1}$ (high) followed by frame-by-frame motion correction for removing

819 movement artifacts associated with respiration and head-unrestrained behavior. The mean image

820 over the imaging session was computed, and the $\mathrm{dF} / \mathrm{F}$ was computed using this mean image. The

821 resultant preprocessed movies were then exported into MATLAB, and cell segmentation was

822 performed using an open-source calcium imaging software (CIAPKG) (Corder et al., 2019).

823 Specifically, we used a Principal Component Analysis/Independent Component Analysis

824 (PCA/ICA) approach to detect and extract ROIs (presumed neurons) per field of view (Mukamel

825 et al., 2009). For each movie, the extracted output neurons were then manually sorted to remove

826 overlapping neurons, neurons with low SNR, and neurons with aberrant shapes.

827 Accepted neurons and their $\mathrm{Ca}^{2+}$ activity traces were exported to MATLAB for further

828 analysis using custom scripts (as previously described in (Frost et al., 2020)). Briefly, we

829 calculated the standard deviation $(\sigma)$ of the $\mathrm{Ca}^{2+}$ movie and used this to perform threshold-based

830 event detection on the traces by first detecting increases in $\mathrm{dF} / \mathrm{F}$ exceeding $2 \sigma$ (over one second).

831 Subsequently, we detected events that exceeded $10 \sigma$ for over two seconds and had a total area

832 under the curve higher than $150 \sigma$. The peak of the event was estimated as the local maximum of

833 the entire event. For an extracted output neuron, active frames were marked as the period from

834 the beginning of an event until the $\mathrm{Ca}^{2+}$ signal decreased $30 \%$ from the peak of the event (up to a

835 maximum of 2 seconds).

837 Procedure for measuring object-related changes in $\mathrm{Ca}^{2+}$ activity

838 Frame-by-frame $\mathrm{x}-\mathrm{y}$ positions of the head of a mouse in the testing cage were detected using

839 DeepLabCut. A small rectangular area surrounding the object location in the testing cage was

840 marked as the object zone. Time points (frames acquired at $30 \mathrm{~Hz}$ and resampled at $20 \mathrm{~Hz}$, using

841 resample function in MATLAB) when the mouse's head was inside the object zone were

842 classified as INbin and the remaining frames were classified as OUTbin. We then recorded the

843 frame-by-frame $\mathrm{Ca}^{2+}$ activity of neurons corresponding to the INbin and OUTbin position 
844 frames. For all extracted neurons, the mean activity for INbin $\left(\mu_{\mathrm{INbin}}\right)$ and OUTbin $\left(\mu_{\mathrm{OUTbin}}\right)$

845 frames were calculated. We also calculated the standard deviation ( $\left.\mathrm{SD}_{\text {OUTbin }}\right)$ of neuronal activity

846 in OUTbin frames. The z-scored activity of each neuron was estimated using equation 2.1. The

847 object signal-to-noise ratio (Object SNR) was calculated using the z-scored activity during HC

848 and NOE epochs (Equation 2.2).

$$
\text { zscore }=\frac{\mu_{\text {INbin }}-\mu_{\text {OUTbin }}}{S D_{\text {OUTbin }}}
$$

$$
\text { Object SNR = } \text { zscore }_{N O E}-\text { Zscore }_{H C}
$$

\section{Histological processing}

\section{Assessment of virus expression and anterograde tracing of LRG projections}

854 Animals were transcardially perfused with PBS, and then with 4\% paraformaldehyde (PFA). The 855 brains were post-fixed for at least one day in PFA solution. Coronal sections (50-75 $\mu \mathrm{m}$ thick) 856 were obtained using a vibratome. Sections that included the injection sites, electrode 857 implantation sites and lens implantation sites were mounted on slides and cover-slipped using a 858 glycerol-base, aqueous mounting medium (Vectashield Plus Antifade Mounting Medium, Vector 859 labs). Sections were first scanned using an upright wide-field fluorescence microscope. 860 Following this, confocal images were taken with 10x and 20x objectives on an Andor Borealis 861 CSU-W1 spinning disk confocal mounted on a Nikon Ti Microscope (UCSF Nikon Imaging 862 Center, NIH S10 Shared Instrumentation grant 1S10OD017993-01A1) and captured with an 863 Andor Zyla sCMOS camera and Micro-Manager software (Open Imaging).

\section{Inhibitory neuron marker expression in recipient CAl neurons}

866 Slices containing biocytin-filled cells were fixed overnight in a buffered solution containing $4 \%$

867 PFA. Slices were rinsed in PBS, then blocked and permeabilized in PBS with 5\% Donkey 868 Serum, $0.3 \%$ Triton X-100 and 1\% BSA. Slices were immuno-stained overnight with one or two 869 primary antibodies: rabbit anti-PV (Swant; diluted 1:200), rat anti-SST (Millipore, diluted 870 1:200), or rabbit anti-VIP (Immunostar, diluted 1:200). Slices were washed 6 x 10min in PBS 871 containing $0.3 \%$ Triton X-100. Slices were incubated with donkey anti-rabbit Alexa-488, donkey 872 anti-rat Alexa 594 secondary antibody (1:800, Thermo Fisher), and Streptavidin-647 (1:300, 873 Thermo Fisher) overnight at $4^{\circ} \mathrm{C}$. After washing $6 \times 10 \mathrm{~min}$ in PBS with $0.3 \%$ Triton X-100, 874 slices were mounted with an aqueous mounting medium. Confocal mages were obtained as 875 described above.

877 Inhibitory neuron (IN) marker expression in CTb tagged PFC LRG neurons

878 5-7 days after CTb injection, mice were transcardially perfused with PBS followed by 4\% PFA 
879 solution, and brains were post-fixed for at least one day. Coronal sections $(75 \mu \mathrm{m})$ were obtained

880 using a vibratome, and immuhistochemistry was performed (as described above). The following

881 primary antibodies were used to stain for IN markers: rabbit anti-PV (Swant; diluted 1:200); rat

882 anti-SST (Millipore, diluted 1:200); rabbit anti-VIP (Immunostar, diluted 1:200); rabbit anti-

883 NPY (Immunostar, diluted 1:500); rabbit anti-calretinin (Immunostar, diluted 1:500); rabbit anti-

884 nNOS (Life technologies, diluted 1:500), and goat anti-CTb (List, diluted 1:500). The following

885 secondary antibodies were used: donkey anti-rabbit Alexa 488; donkey anti-rat Alexa 488; and

886 donkey anti-goat Alexa 594. For each IN marker, confocal images collected from mounted

887 sections were used to manually count the number of $\mathrm{CTb}+$ and IN marker+ PFC neurons (ImageJ

888 software).

\section{Statistical analysis}

891 Detailed statistical analyses were performed using MATLAB and Graphpad Prism. Comparisons

892 of means were performed using paired or unpaired two-tailed Student's t test, one-way ANOVA 893 or two-way repeated measures ANOVA with Tukey post hoc test unless otherwise stated. For

894 non-parametric data sets, we used a Chi-square test to determine significance. Sample sizes and 895 statistical tests and parameters are listed in the figure legends. Data are reported as mean \pm 896 S.E.M. unless otherwise stated.

Data availability

899 Data supporting the findings of this study are available from the corresponding author upon

900 reasonable request.

901

\section{Code availability}

903 Custom code used in this study is available from the corresponding author upon reasonable 904 request. 


\section{SUPPLEMENTAL INFORMATION: FIGURES AND LEGENDS}
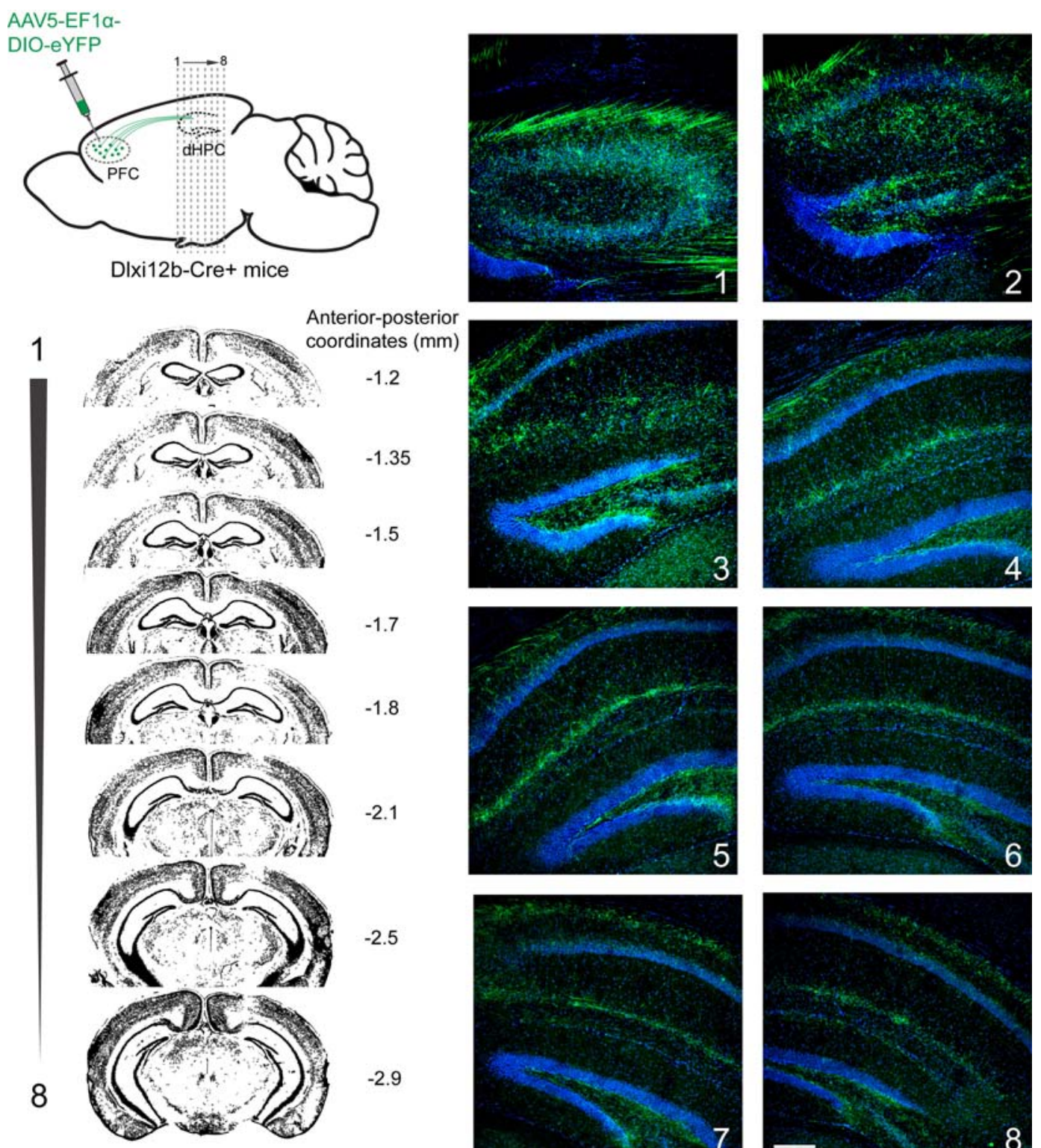

$-1.7$

$-1.8$

$-2.1$

ates ( $\mathrm{mm}$ )

$-1.35$

$-1.5$

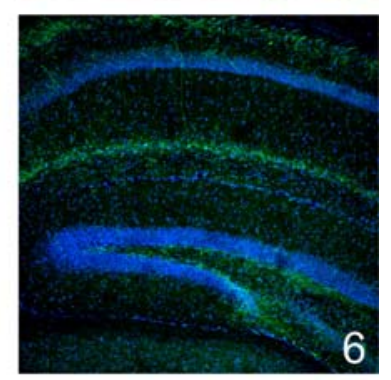

$-2.5$
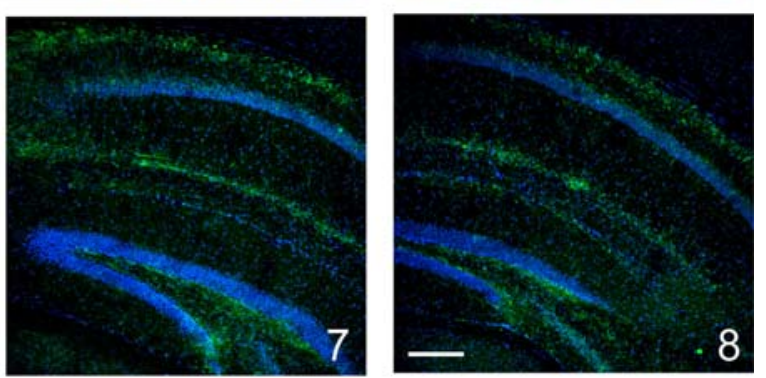

905 Figure S1, related to Figure 1: Anterograde tracing of HPC projecting PFC LRG neurons.

906 Left: Schematic illustrating the anterograde tracing strategy. Microinjection of Cre-dependent

907 eYFP virus into the PFC of Dlxi12b-Cre+ mice. Coronal slices of the hippocampus were

908 obtained at increasing anterior-posterior (AP) distance from Bregma. Right: DAPI stained

909 hippocampal sections showing eYFP+ PFC LRG axon terminals (green). Numbers on the right

910 indicate the anterior-posterior (AP) coordinates w.r.t. bregma. Scale bar, $200 \mu \mathrm{m}$. 
A

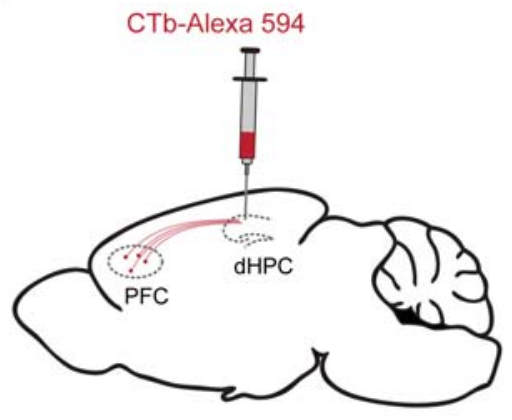

C

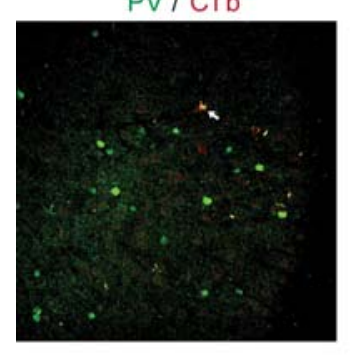

$\mathrm{CR} / \mathrm{CTb}$

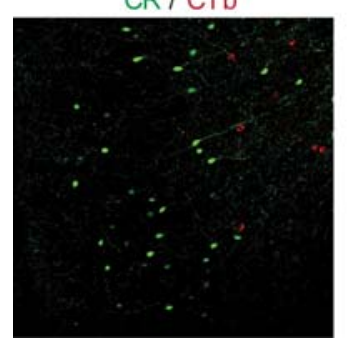

B

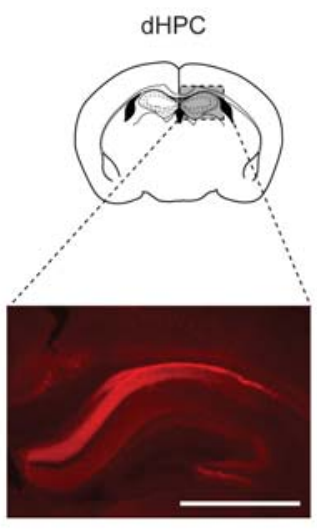

VIP / CTb

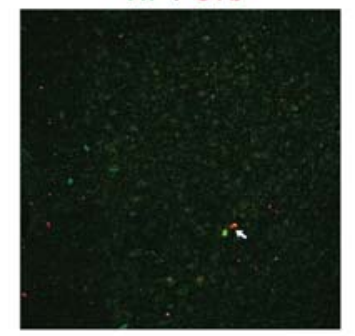

nNOS / CTb

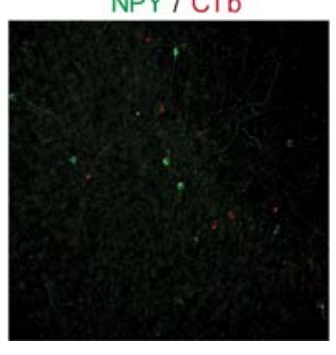

SST / CTb
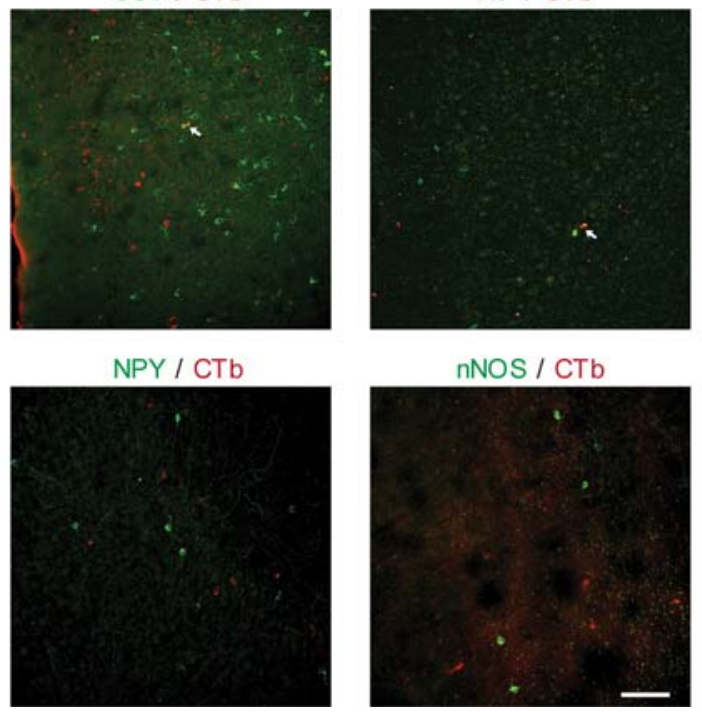

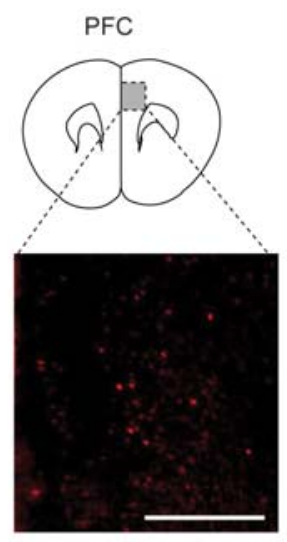

D

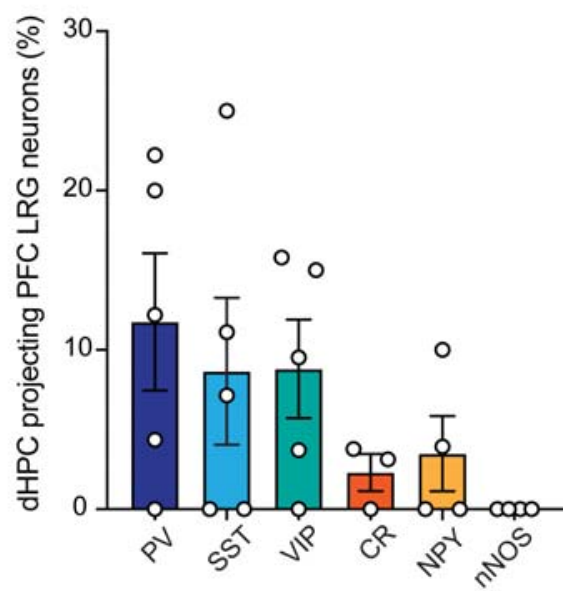

911 Figure S2, related to Figure 1: Inhibitory neuron marker expression in dHPC projecting 912 PFC LRG neurons.

913 (A) Schematic illustrating the experimental design: Retrograde tracer, Alexa 594 conjugated 914 Cholera Toxin (CTb), was injected into dHPC.

915 (B) Representative images showing Alexa 594-CTb injection site in dHPC section (left) and

$916 \mathrm{CTb}+$ neurons in PFC (right). Note: there is a high density of labeled axons in the corpus 917 callosum. Scale bar, $1 \mathrm{~mm}$ (left) and $250 \mu \mathrm{m}$ (right).

918 (C) Example images showing co-labeling of inhibitory neuron markers (green) in CTb+ dHPC919 projecting PFC LRG neurons. Parvalbumin (PV), Somatostatin (SST), Vasoactive intestinal 920 polypeptide (VIP), Calretinin (CR), Neuropeptide Y (NPY), neuronal nitric oxide synthase 921 (nNOS). Scale bar, $100 \mu \mathrm{m}$.

922 (D) Percentage CTb+ dHPC projecting PFC LRG neurons co-expressing various inhibitory 923 neuron markers (mean \pm SEM). Empty circles represent values from individual sections. 
A

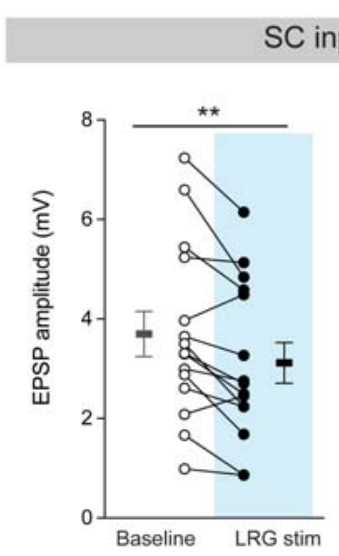

C

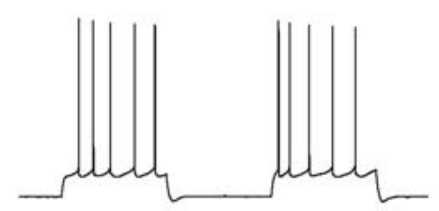

Recipient CA1 interneuron

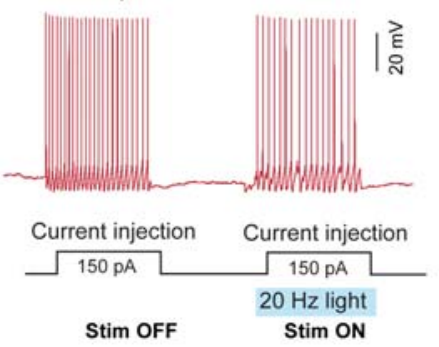

B

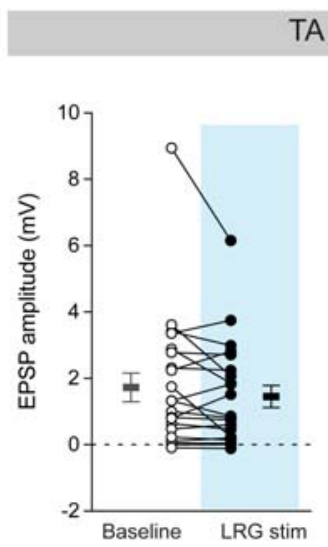

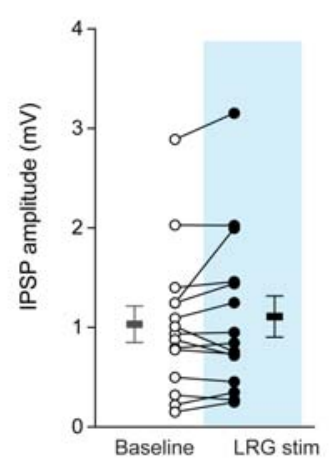

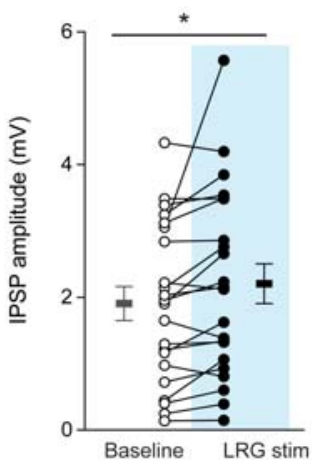

D
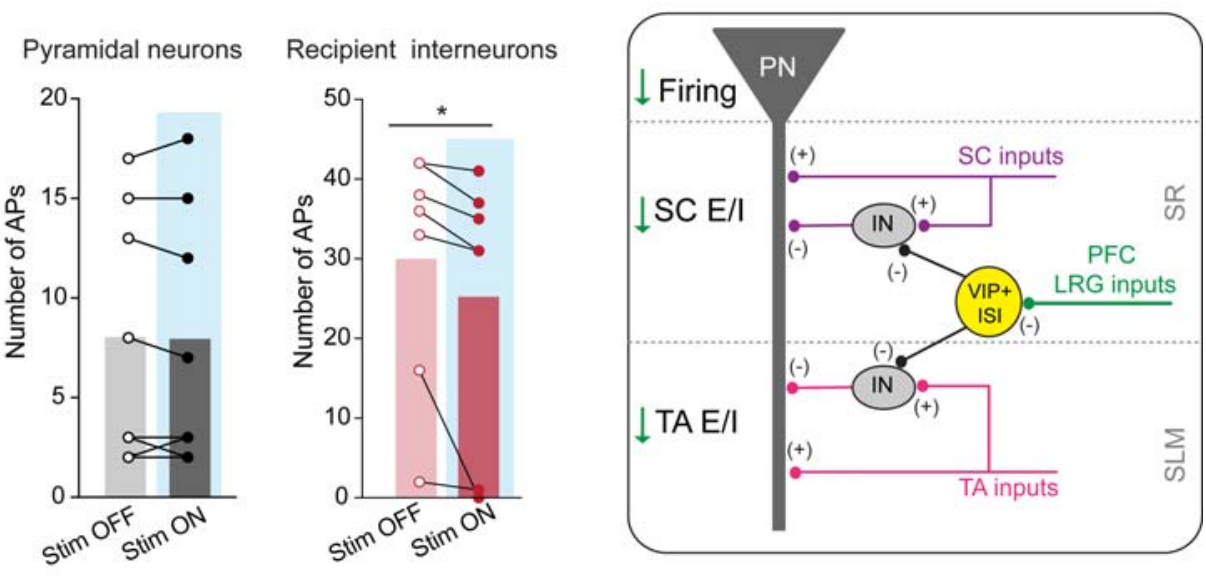

Figure S3, related to Figure 2: Dorsal HPC projecting PFC LRG neurons increase afferent input mediated feedforward inhibition in the CA1 microcircuit.

926 (A) Amplitudes of SC (Schaffer collateral) pathway mediated EPSPs (left) and IPSPs (right) recorded in CA1 pyramidal neurons with (LRG stim) and without (Baseline) concomitant optogenetic stimulation of PFC LRG projections. Open and filled circles represent individual neurons $(\mathrm{n}=15)$, horizontal lines represent averages $( \pm \mathrm{SEM})$. Paired two-way t-test, ** $\mathrm{p}<$ $930 \quad 0.01$.

931 (B) Same as A for TA (temporoammonic) input pathway mediated EPSPs and IPSPs $(\mathrm{n}=22)$.

932 Paired two-way t-test, $* \mathrm{p}<0.05$.

933 (C) Left: example voltage traces showing firing in response to depolarizing current injections

934 with (Stim ON) and without (Stim OFF) concomitant optogenetic stimulation of PFC-dHPC

935 LRG projections (470 nm, $5 \mathrm{~ms}$ pulses at $20 \mathrm{~Hz}$; blue bar). Black trace is an example voltage

936 response in CA1 pyramidal neuron. Red trace shows example voltage response in recipient CA1

937 interneuron. Right: firing output in the absence of optogenetic stimulation (Stim OFF) and during 
938 concomitant optogenetic stimulation $($ Stim ON) in CA1 pyramidal neurons $(\mathrm{n}=10)$ and recipient

939 CA1 interneurons $(n=7)$. Open and filled circles represent individual neurons, bars represent 940 the average values. Two-way paired t-test; $* \mathrm{p}<0.05$.

941 (E) Schematic illustrating the effects of PFC-dHPC LRG projection activation on microcircuit

942 computation in dorsal CA1. PFC LRG projections inhibit VIP+ disinhibitory interneurons, and

943 thereby increase SC and TA pathway mediated feedforward inhibition onto CA1 pyramidal

944 neurons. The reduction in excitation-inhibition ratio at the afferent inputs reduces the afferent

945 input mediated firing of CA1 pyramidal neurons. 
A

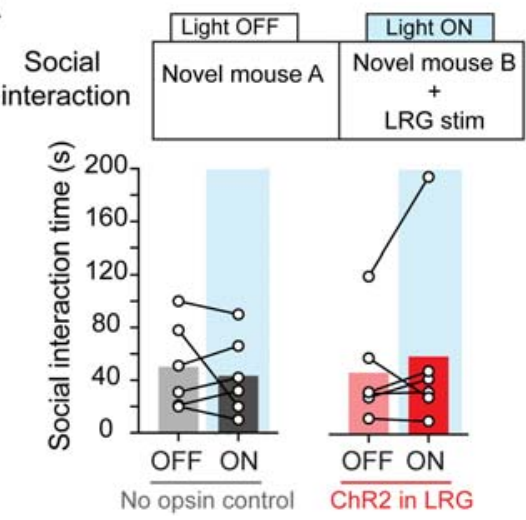

B

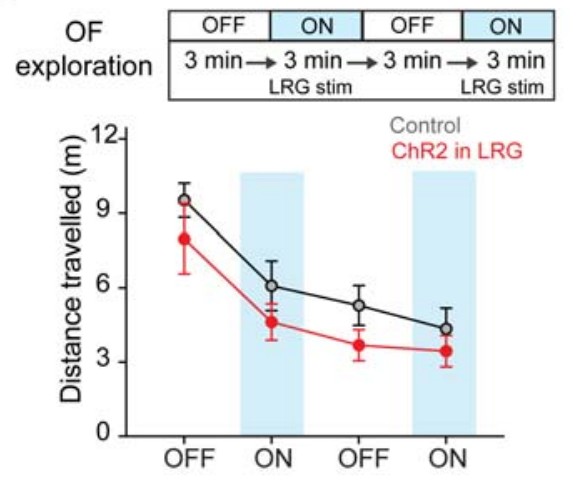

C

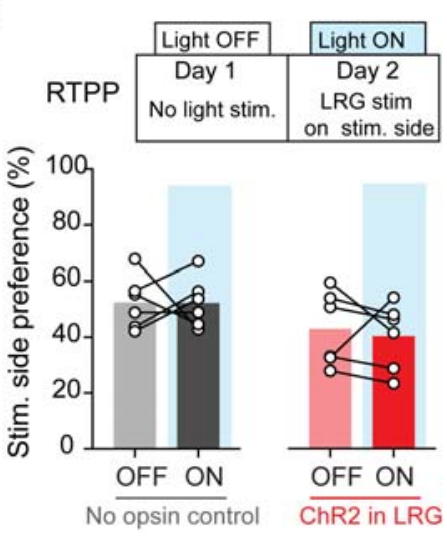

946 Figure S4 related to Figure 3: Optogenetic stimulation of PFC-dHPC LRG projections 947 during social and exploratory behaviors.

948 (A) Top: mice were tested for social interaction behavior with and without optogenetic 949 stimulation. Bottom: Time spent interacting with a novel juvenile mouse is plotted for control 950 and ChR2-expressing mice during light OFF and light ON periods. Open circles represent data 951 from individual mice and bars represent the average values.

952 (B) Top: During open-field (OF) exploration, PFC-dHPC LRG projections were optogenetically 953 stimulated during the second and fourth 3-min epochs of the testing session. Bottom: Average ( \pm 954 SEM) distance travelled during OF exploration during light ON and light OFF epochs is plotted 955 for control and ChR2-expressing mice.

956 (C) Top: experimental design for real-time place preference (RTPP) test. Bottom: Preference for 957 the stimulated chamber during light OFF and light ON conditions (expressed as a \% of the total 958 time) is plotted for control and ChR2-expressing mice. Open circles represent data from 959 individual mice and bars represent the average values. 
A

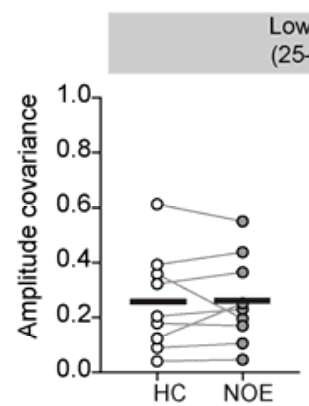

Low- $\gamma$ band

$5-55 \mathrm{~Hz})$

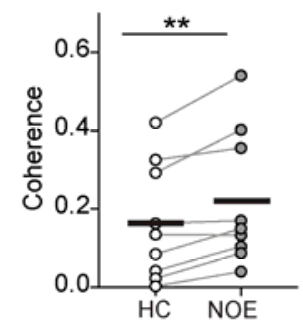

B

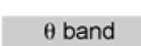

$(4-12 \mathrm{~Hz})$
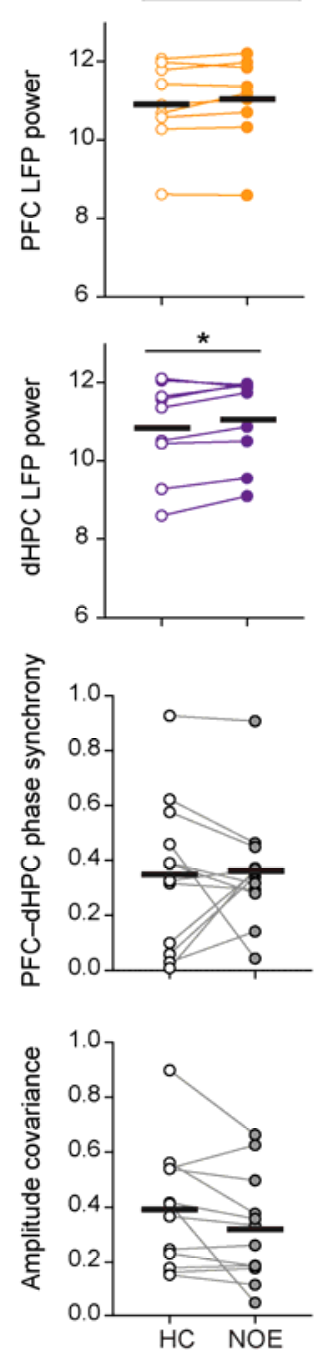
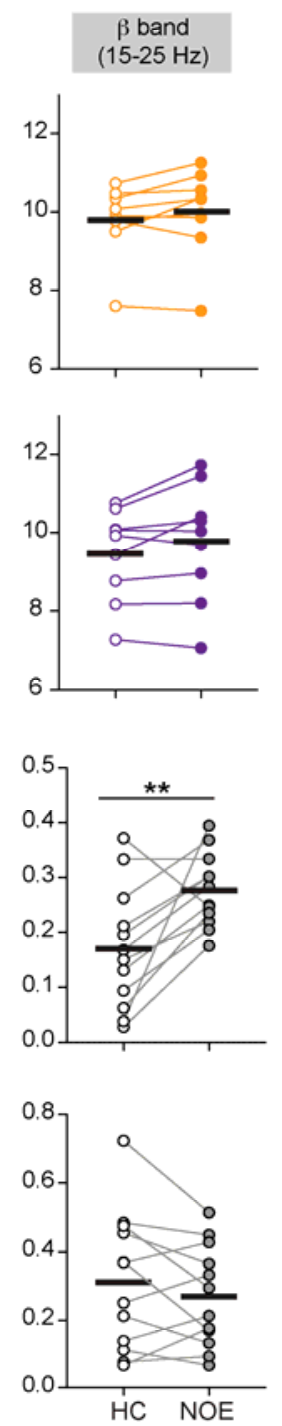

C

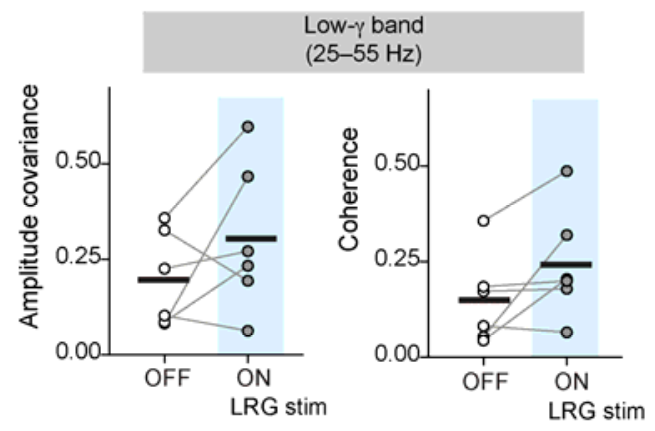

D
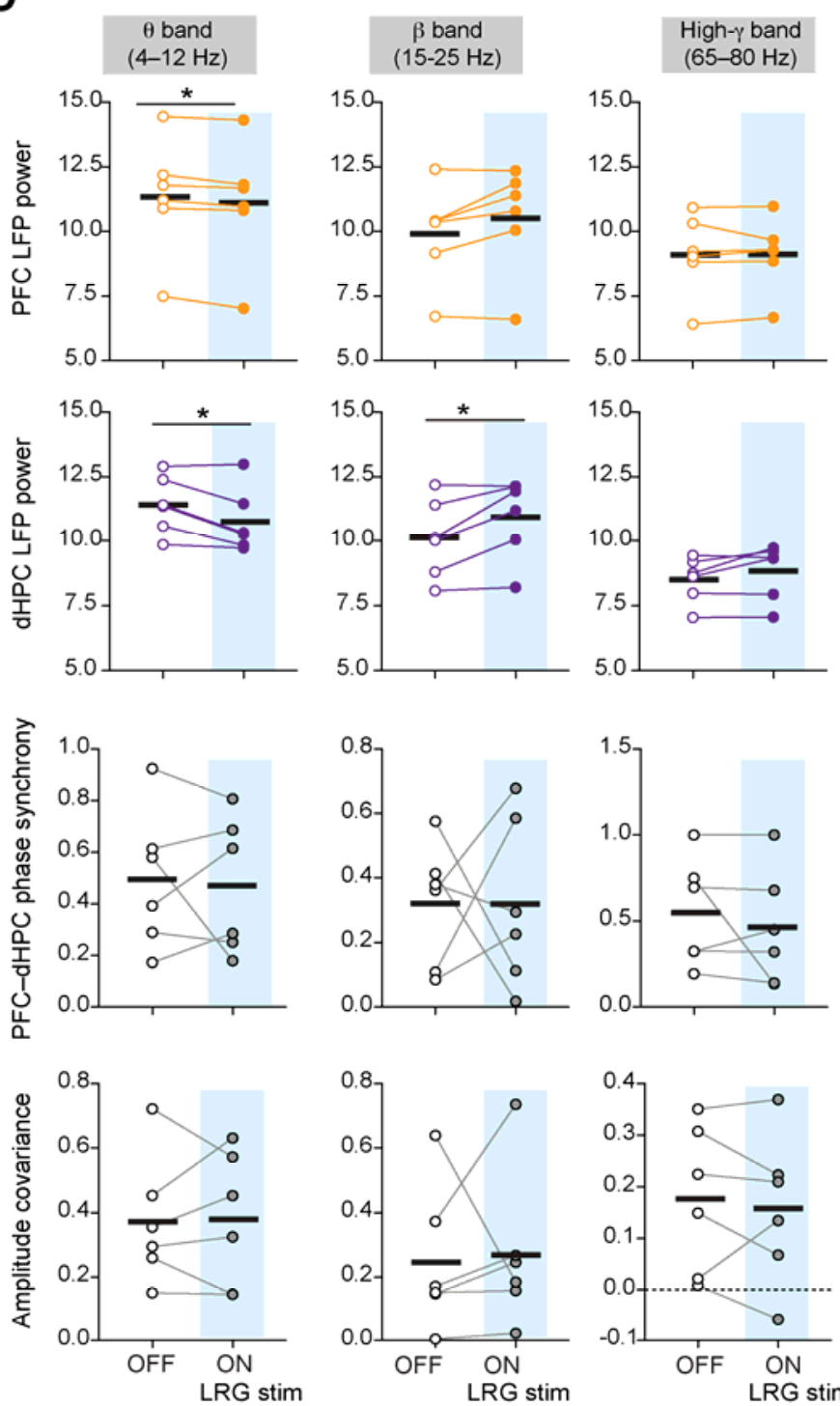

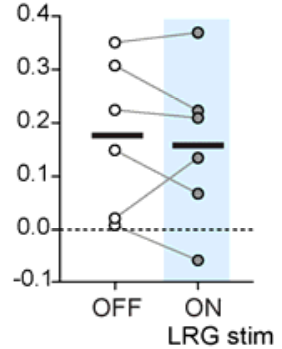

960 Figure S5 related to Figure 4: Effect of optogenetic stimulation of PFC-dHPC LRG 961 projections and NOE on oscillatory activity in dHPC and PFC.

962 (A) Low-gamma (low- $\gamma$ ) amplitude covariance and coherence between PFC and dHPC LFPs

963 recorded during home cage exploration (HC) and novel object exploration (NOE) epochs. Open 
964 and filled circles represent data from individual mice $(\mathrm{n}=9)$ and solid black lines represent

965 average values. Two-way paired t-test, ** $\mathrm{p}<0.01$.

966 (B) PFC and dHPC LFP log power, PFC-dHPC phase synchrony, and PFC-dHPC amplitude

967 covariance for the theta $(\theta)$, beta $(\beta)$ and high gamma (high- $\gamma$ ) frequency bands during $\mathrm{HC}$ and

968 NOE epochs are plotted. Open and filled circles represent data from individual mice $(n=9)$ and

969 solid black lines represent average values. Two-way paired t-test; $* \mathrm{p}<0.05, * * \mathrm{p}<0.01$.

970 (C) Low- $\gamma$ amplitude covariance and coherence between PFC and dHPC LFPs recorded during 971 Light OFF and Light ON epochs (optogenetic stimulation; $473 \mathrm{~nm}, 5 \mathrm{~ms}$ pulses at $20 \mathrm{~Hz}$; blue 972 bars). Open and filled circles represent data from individual mice $(n=6)$ and solid black lines 973 represent average values.

974 (D) PFC and dHPC LFP log power, PFC-dHPC phase synchrony, and PFC-dHPC amplitude 975 covariance for the theta $(\theta)$, beta $(\beta)$ and high-gamma (high- $\gamma$ ) frequency bands during Light $976 \mathrm{OFF}$ and Light $\mathrm{ON}$ epochs are plotted. Open and filled circles represent data from individual 977 mice $(n=6)$ and solid black lines represent average values. Two-way paired $\mathrm{t}$-test; $* \mathrm{p}<0.05$. 

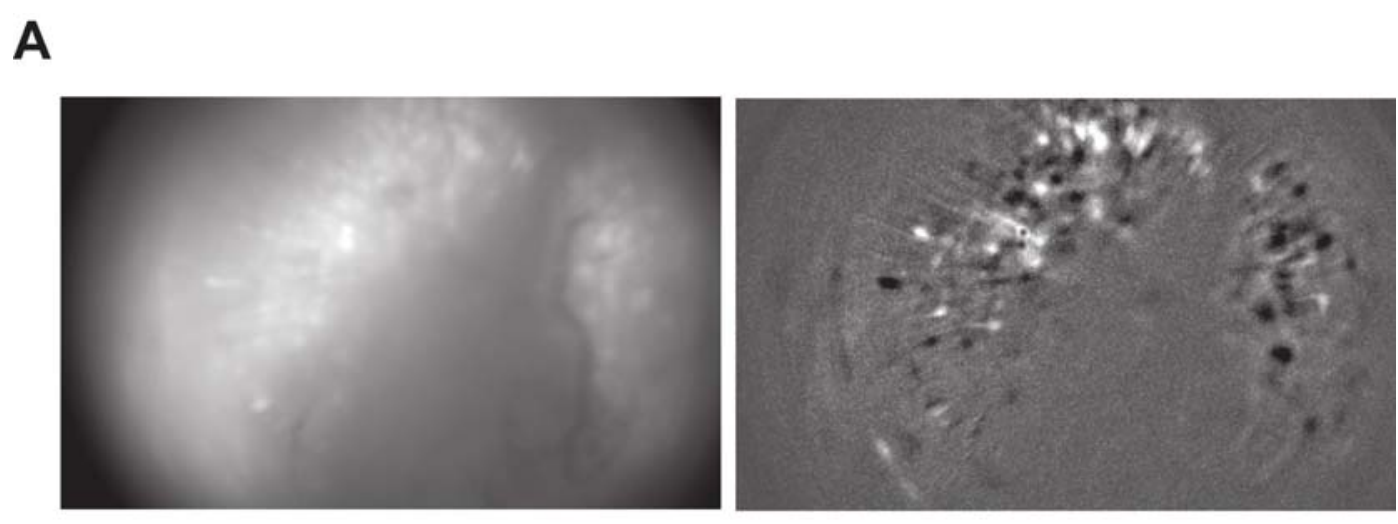

B

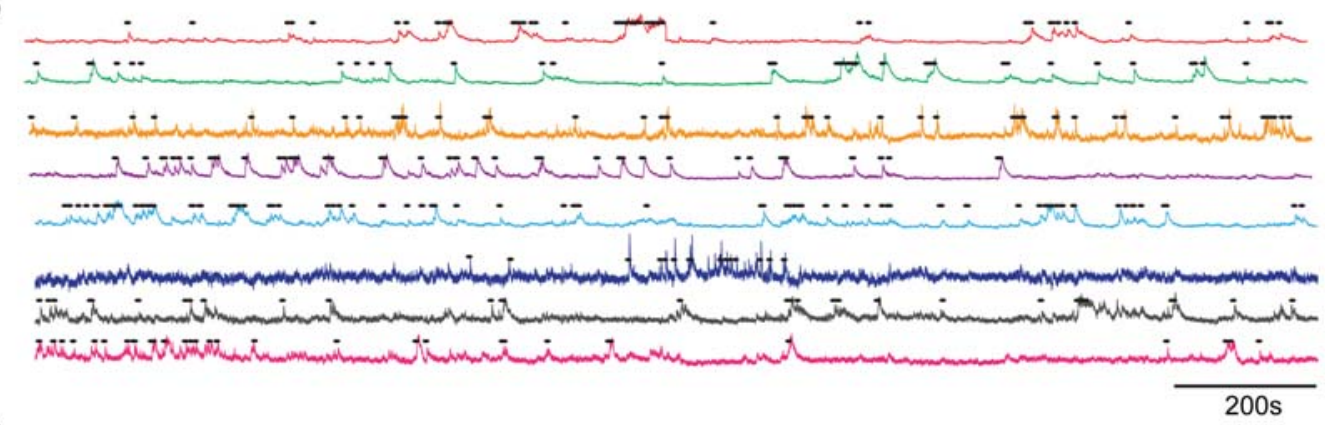

C

Day 1 NOE effect

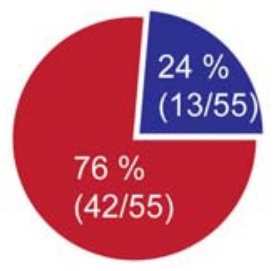

Day 2 LRG stim effect

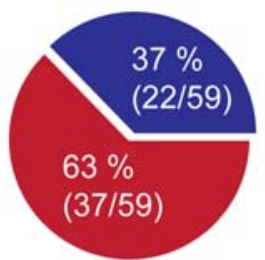

Day 2

LRG stim + NOE effect

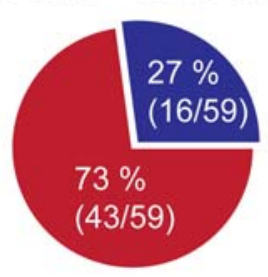

Increased event rate Decreased event rate

978 Figure S6, related to Figure 5: In vivo $\mathrm{Ca}^{2+}$ activity of dorsal CA1 neurons during 979 optogenetic stimulation of PFC LRG projections.

980 (A) Left: Representative raw epifluorescence image showing jGCaMP7f expression in the dorsal

981 CA1 imaged using a miniaturized microscope in a freely behaving mouse. Right: Transformed 982 image showing relative change in fluorescence $(\mathrm{dF} / \mathrm{F})$.

983 (B) Representative $\mathrm{Ca}^{2+}$ transients (colored lines) recorded from dorsal CA1 neurons. Black dots 984 above the traces represent the detected active $\mathrm{Ca}^{2+}$ events.

985 (C) Pie charts showing the fraction of CA1 neurons that decrease or increase activity during 986 NOE on day 1 testing, during LRG stimulation on day 2 testing, or during NOE in the presence 987 of LRG stimulation on day 2 testing. 


\section{SUPPLEMENTAL INFORMATION: TABLES}

Table S1, related to Figure 1: Electrophysiological heterogeneity of dHPC projecting PFC

\section{LRG neurons.}

990 Mean ( \pm SEM) values for resting membrane properties, action-potential (AP) properties, and 991 firing properties of dHPC projecting PFC LRG neurons classified as regular spiking, fast 992 spiking, and irregular spiking (Fig. 1H). AHP, afterhyperpolarization; SFA, spike frequency 993 accommodation.

\begin{tabular}{|c|c|c|c|}
\hline $\begin{array}{c}\text { Electrophysiological } \\
\text { property }\end{array}$ & $\begin{array}{c}\text { Regular spiking } \\
\qquad \mathrm{n}=9\end{array}$ & $\begin{array}{c}\text { Fast spiking } \\
\qquad \mathrm{n}=4\end{array}$ & $\begin{array}{c}\text { Irregular } \\
\text { spiking } \\
n=3\end{array}$ \\
\hline $\begin{array}{l}\text { Resting membrane potential } \\
\qquad(\mathrm{mV})\end{array}$ & $-71 \pm 1.1$ & $-65.5 \pm 2.7$ & $-67.6 \pm 1.6$ \\
\hline Input Resistance $(\mathrm{M} \Omega)$ & $256.2 \pm 25.4$ & $171 \pm 47.2$ & $383.3 \pm 75.3$ \\
\hline Membrane time constant (ms) & $15.1 \pm 2.3$ & $11.3 \pm 2.1$ & $20.9 \pm 4.6$ \\
\hline Sag ratio & $1.01 \pm 0.07$ & $1.03 \pm 0.02$ & $1.05 \pm 0.05$ \\
\hline Rebound slope (mV) & $-0.04 \pm 0.01$ & $-0.04 \pm 0.02$ & $-0.4 \pm 0.41$ \\
\hline Rheobase (pA) & $113.8 \pm 18.6$ & $162.5 \pm 54.5$ & $50 \pm 25$ \\
\hline AP amplitude (mV) & $75.3 \pm 4.4$ & $86.5 \pm 4$ & $93 \pm 2.2$ \\
\hline AP rate of rise $(\mathrm{mV} / \mathrm{s})$ & $308.2 \pm 32.2$ & $468.4 \pm 67.4$ & $316.8 \pm 51.9$ \\
\hline AP threshold (mV) & $-41.6 \pm 1$ & $-50.6 \pm 1.7$ & $-47.7 \pm 1.2$ \\
\hline AP half-width (ms) & $0.74 \pm 0.07$ & $0.37 \pm 0.04$ & $0.84 \pm 0.19$ \\
\hline Fast AHP (mV) & $13.7 \pm 0.3$ & $19.1 \pm 0.4$ & $13.5 \pm 1.2$ \\
\hline SFA index & $2.5 \pm 0.2$ & $1.5 \pm 0.1$ & $5.4 \pm 0.5$ \\
\hline Max. firing frequency $(\mathrm{Hz})$ & $46 \pm 4.8$ & $117.9 \pm 11.5$ & 16.81 .53 \\
\hline
\end{tabular}


994 Table S2, related to Figure 2: Electrophysiological properties of dHPC interneurons receiving 995 direct PFC LRG inputs.

996 Mean ( \pm SEM) values for resting membrane properties, action-potential (AP) properties, firing 997 properties, and optogenetically evoked postsynaptic current (oPSC) amplitudes recorded from recipient 998 CA1 interneurons classified as regular spiking, fast spiking, and irregular spiking (Fig. 2B).

999 AHP, afterhyperpolarization; SFA, spike frequency accommodation.

\begin{tabular}{|c|c|c|c|}
\hline $\begin{array}{c}\text { Electrophysiological } \\
\text { property }\end{array}$ & $\begin{array}{c}\text { Regular spiking } \\
\mathrm{n}=25\end{array}$ & $\begin{array}{c}\text { Fast spiking } \\
\mathrm{n}=11\end{array}$ & $\begin{array}{c}\text { Irregular spiking } \\
\qquad \mathrm{n}=19\end{array}$ \\
\hline $\begin{array}{l}\text { Resting membrane potential } \\
\qquad(\mathrm{mV})\end{array}$ & $-65 \pm 2.1$ & $-68.3 \pm 1.3$ & $-66.8 \pm 1.2$ \\
\hline Input Resistance $(\mathrm{M} \Omega)$ & $218.2 \pm 18.2$ & $248.2 \pm 27.2$ & $235.4 \pm 21.9$ \\
\hline Rheobase (pA) & $105 \pm 15.3$ & $77.2 \pm 30.2$ & $100 \pm 15.6$ \\
\hline AP amplitude (mV) & $84.2 \pm 2.7$ & $83.1 \pm 2.8$ & $85.5 \pm 2.07$ \\
\hline $\mathrm{AP}$ rate of rise $(\mathrm{mV} / \mathrm{s})$ & $386.9 \pm 20.9$ & $376.5 \pm 25.9$ & $358 \pm 17.1$ \\
\hline AP threshold $(\mathrm{mV})$ & $-46.1 \pm 0.8$ & $-47.5 \pm 1.4$ & $-46.9 \pm 1$ \\
\hline AP half-width (ms) & $0.58 \pm 0.03$ & $0.43 \pm 0.03$ & $0.54 \pm 0.02$ \\
\hline Fast AHP $(\mathrm{mV})$ & $10.1 \pm 1$ & $16.3 \pm 1.35$ & $13.5 \pm 0.9$ \\
\hline SFA index & $3.5 \pm 0.3$ & $2.2 \pm 0.26$ & $5.9 \pm 0.7$ \\
\hline Max. firing frequency $(\mathrm{Hz})$ & $53.7 \pm 2.44$ & $127.5 \pm 8.1$ & $50.4 \pm 8.5$ \\
\hline oPSC amplitude (pA) & $324.3 \pm 53.2$ & $193.4 \pm 47.3$ & $172.3 \pm 25.3$ \\
\hline
\end{tabular}




\section{REFERENCES}

1000 Acsády, L., Arabadzisz, D., and Freund, T.F. (1996a). Correlated morphological and neurochemical 1001 features identify different subsets of vasoactive intestinal polypeptide-immunoreactive interneurons in 1002 rat hippocampus. Neuroscience 73, 299-315.

1003 Acsády, L., Görcs, T.J., and Freund, T.F. (1996b). Different populations of vasoactive intestinal 1004 polypeptide-immunoreactive interneurons are specialized to control pyramidal cells or interneurons in 1005 the hippocampus. Neuroscience 73, 317-334.

1006 Ang, C.W., Carlson, G.C., and Coulter, D.A. (2005). Hippocampal CA1 Circuitry Dynamically Gates 1007 Direct Cortical Inputs Preferentially at Theta Frequencies. J Neurosci 25, 9567-9580.

1008 Basu, J., Zaremba, J.D., Cheung, S.K., Hitti, F.L., Zemelman, B.V., Losonczy, A., and Siegelbaum, S.A. 1009 (2016). Gating of hippocampal activity, plasticity, and memory by entorhinal cortex long-range 1010 inhibition. Science 351, aaa5694-aaa5694.

1011 Bähner, F., Demanuele, C., Schweiger, J., Gerchen, M.F., Zamoscik, V., Ueltzhöffer, K., Hahn, T., 1012 Meyer, P., Flor, H., Durstewitz, D., et al. (2015). Hippocampal-dorsolateral prefrontal coupling as a 1013 species-conserved cognitive mechanism: a human translational imaging study.

1014 Neuropsychopharmacology 40, 1674-1681.

1015 Bittner, K.C., Grienberger, C., Vaidya, S.P., Milstein, A.D., Macklin, J.J., Suh, J., Tonegawa, S., and 1016 Magee, J.C. (2015). Conjunctive input processing drives feature selectivity in hippocampal CA1 1017 neurons. Nat Neurosci 18, 1133-1142.

1018 Brincat, S.L., and Miller, E.K. (2015). Frequency-specific hippocampal-prefrontal interactions during 1019 associative learning. Nat Neurosci 18, 576-581.

1020 Chamberland, S., and Topolnik, L. (2012). Inhibitory control of hippocampal inhibitory neurons. Front. 1021 Neurosci. 6, 165.

1022 Chattopadhyaya, B., and Cristo, G.D. (2012). GABAergic circuit dysfunctions in neurodevelopmental 1023 disorders. Front Psychiatry 3, 51-51.

1024 Christenson Wick, Z., Tetzlaff, M.R., and Krook-Magnuson, E. (2019). Novel long-range inhibitory 1025 nNOS-expressing hippocampal cells. eLife 8, e46816.

1026

1027

1028

1029

1030

1031 1032

1033 1034
Churchwell, J.C., Morris, A.M., Musso, N.D., and Kesner, R.P. (2010). Prefrontal and hippocampal contributions to encoding and retrieval of spatial memory. Neurobiology of Learning and Memory 93, 415-421.

Colgin, L.L. (2011). Oscillations and hippocampal-prefrontal synchrony. Current Opinion in Neurobiology 21, 467-474.

Corder, G., Ahanonu, B., Grewe, B.F., Wang, D., Schnitzer, M.J., and Scherrer, G. (2019). An amygdalar neural ensemble that encodes the unpleasantness of pain. Science 363, 276.

Csicsvari, J., Jamieson, B., Wise, K.D., and Buzsáki, G. (2003). Mechanisms of Gamma Oscillations in the Hippocampus of the Behaving Rat. Neuron 37, 311-322. 
1035 Cunha-Reis, D., and Caulino-Rocha, A. (2020). VIP Modulation of Hippocampal Synaptic Plasticity: A 1036 Role for VIP Receptors as Therapeutic Targets in Cognitive Decline and Mesial Temporal Lobe 1037 Epilepsy. Front Cell Neurosci 14, 153-153.

1038

1039

1040

1041

1042

1043

1044

1045

1046

1047

1048

1049

1050

1051

1052

1053

1054

1055

1056

1057

1058

1059

1060

1061

1062

1063

1064

1065

1066

1067

1068 1069

1070

1071

1072
Cunniff, M.M., Markenscoff-Papadimitriou, E., Ostrowski, J., Rubenstein, J.L., and Sohal, V.S. (2020). Altered hippocampal-prefrontal communication during anxiety-related avoidance in mice deficient for the autism-associated gene Pogz. eLife 9, e54835.

Dana, H., Sun, Y., Mohar, B., Hulse, B.K., Kerlin, A.M., Hasseman, J.P., Tsegaye, G., Tsang, A., Wong, A., Patel, R., et al. (2019). High-performance calcium sensors for imaging activity in neuronal populations and microcompartments. Nat Meth 16, 649-657.

del Rio, D., Beucher, B., Lavigne, M., Wehbi, A., Gonzalez Dopeso-Reyes, I., Saggio, I., and Kremer, E.J. (2019). CAV-2 Vector Development and Gene Transfer in the Central and Peripheral Nervous Systems. Front. Mol. Neurosci. 12, 1202.

DeVito, L.M., and Eichenbaum, H. (2010). Distinct contributions of the hippocampus and medial prefrontal cortex to the "what-where-when" components of episodic-like memory in mice. Behav Brain Res 215, 318-325.

Eichenbaum, H. (2017). Prefrontal-hippocampal interactions in episodic memory. Nat Rev Neurosci 18, 547-558.

Fanselow, M.S., and Dong, H.-W. (2010). Are the Dorsal and Ventral Hippocampus Functionally Distinct Structures? Neuron 65, 7-19.

Floresco, S.B., Seamans, J.K., and Phillips, A.G. (1997). Selective Roles for Hippocampal, Prefrontal Cortical, and Ventral Striatal Circuits in Radial-Arm Maze Tasks With or Without a Delay. J Neurosci 17,1880 .

Francavilla, R., Villette, V., Luo, X., Chamberland, S., Muñoz-Pino, E., Camiré, O., Wagner, K., Kis, V., Somogyi, P., and Topolnik, L. (2018). Connectivity and network state-dependent recruitment of long-range VIP-GABAergic neurons in the mouse hippocampus. Nature Communications 9, 5043.

Frost, N.A., Haggart, A., and Sohal, V.S. (2020). Dynamic correlations help prefrontal ensembles transmit information about social behavior. bioRxiv 2020.08.05.238741.

Gazzaley, A., and D'Esposito, M. (2007). Unifying prefrontal cortex function: Executive control, neural networks, and top-down modulation. In The Human Frontal Lobes: Functions and Disorders, 2nd Ed, (New York, NY, US: The Guilford Press), pp. 187-206.

Gilbert, S.J., Bird, G., Brindley, R., Frith, C.D., and Burgess, P.W. (2008). Atypical recruitment of medial prefrontal cortex in autism spectrum disorders: An fMRI study of two executive function tasks. Neuropsychologia 46, 2281-2291.

Godsil, B.P., Kiss, J.P., Spedding, M., and Jay, T.M. (2013). The hippocampal-prefrontal pathway: the weak link in psychiatric disorders? European Neuropsychopharmacology 23, 1165-1181.

Grienberger, C., Milstein, A.D., Bittner, K.C., Romani, S., and Magee, J.C. (2017). Inhibitory suppression of heterogeneously tuned excitation enhances spatial coding in CA1 place cells. Nat Neurosci 20, 417-426. 
1073 Guise, K.G., and Shapiro, M.L. (2017). Medial Prefrontal Cortex Reduces Memory Interference by 1074 Modifying Hippocampal Encoding. Neuron 94, 183-192.e188.

1075 Hallock, H.L., Wang, A., and Griffin, A.L. (2016). Ventral Midline Thalamus Is Critical for 1076 Hippocampal-Prefrontal Synchrony and Spatial Working Memory. J. Neurosci 36, 8372-8389.

1077 Hare, B.D., and Duman, R.S. (2020). Prefrontal cortex circuits in depression and anxiety: contribution of 1078 discrete neuronal populations and target regions. Molecular Psychiatry 2014 20:7 25, 2742-2758.

1079 Hnasko, T.S., Perez, F.A., Scouras, A.D., Stoll, E.A., Gale, S.D., Luquet, S., Phillips, P.E.M., Kremer, 1080 E.J., and Palmiter, R.D. (2006). Cre recombinase-mediated restoration of nigrostriatal dopamine in 1081 dopamine-deficient mice reverses hypophagia and bradykinesia. Proc Natl Acad Sci USA 103, 8858.

1082 Hoover, W.B., and Vertes, R.P. (2007). Anatomical analysis of afferent projections to the medial 1083 prefrontal cortex in the rat. Brain Struct Funct 212, 149-179.

1084 Hoover, W.B., and Vertes, R.P. (2012). Collateral projections from nucleus reuniens of thalamus to 1085 hippocampus and medial prefrontal cortex in the rat: a single and double retrograde fluorescent labeling 1086 study. Brain Struct Funct 217, 191-209.

Jay, T.R.S.M., and Witter, M.P. (1991). Distribution of hippocampal CA1 and subicular efferents in the 1089 1091 Neurosci. 9, 1082.

1092 1093 1094

1095 1096
1090 Jin, J., and Maren, S. (2015). Prefrontal-Hippocampal Interactions in Memory and Emotion. Front. Syst.

prefrontal cortex of the rat studied by means of anterograde transport ofPhaseolus vulgarisleucoagglutinin. J. Comp. Neurol. 313, 574-586.

Jinno, S., Klausberger, T., Marton, L.F., Dalezios, Y., Roberts, J.D.B., Fuentealba, P., Bushong, E.A., Henze, D., Buzsaki, G., and Somogyi, P. (2007). Neuronal Diversity in GABAergic Long-Range Projections from the Hippocampus. J. Neurosci 27, 8790-8804.

Jones, M.W., and Wilson, M.A. (2005). Theta Rhythms Coordinate Hippocampal-Prefrontal Interactions in a Spatial Memory Task. PLoS Biol 3, e402.

Klapoetke, N.C., Murata, Y., Kim, S.S., Pulver, S.R., Birdsey-Benson, A., Cho, Y.K., Morimoto, T.K., Chuong, A.S., Carpenter, E.J., Tian, Z., et al. (2014). Independent optical excitation of distinct neural populations. Nat Meth 11, 338-346.

Kupferschmidt, D.A., and Gordon, J.A. (2018). The dynamics of disordered dialogue: Prefrontal, hippocampal and thalamic miscommunication underlying working memory deficits in schizophrenia. Brain and Neuroscience Advances 2, 239821281877182.

Kyd, R.J., and Bilkey, D.K. (2003). Prefrontal Cortex Lesions Modify the Spatial Properties of Hippocampal Place Cells. Cerebral Cortex 13, 444-451.

Lee, A.T., Cunniff, M.M., See, J.Z., Wilke, S.A., Luongo, F.J., Ellwood, I.T., Ponnavolu, S., and Sohal, V.S. (2019). VIP Interneurons Contribute to Avoidance Behavior by Regulating Information Flow across Hippocampal-Prefrontal Networks. Neuron 102, 1223-1234.e1224. 
1108 Lee, A.T., Vogt, D., Rubenstein, J.L., and Sohal, V.S. (2014). A Class of GABAergic Neurons in the 1109 Prefrontal Cortex Sends Long-Range Projections to the Nucleus Accumbens and Elicits Acute 1110 Avoidance Behavior. J Neurosci 34, 11519-11525.

1111 Li, M., Long, C., and Yang, L. (2015). Hippocampal-Prefrontal Circuit and Disrupted Functional

1112 Connectivity in Psychiatric and Neurodegenerative Disorders. BioMed Research International 2015, $1113810548-810548$.

1114 Malik, R., and Johnston, D. (2017). Dendritic GIRK Channels Gate the Integration Window, Plateau 1115 Potentials, and Induction of Synaptic Plasticity in Dorsal But Not Ventral CA1 Neurons. J Neurosci 37 , 11163940.

1117 Malik, R., Dougherty, K.A., Parikh, K., Byrne, C., and Johnston, D. (2015). Mapping the 1118 electrophysiological and morphological properties of CA1 pyramidal neurons along the longitudinal 1119 hippocampal axis. Hippocampus 26, 341-361.

1120 Malik, R., Pai, E.L.-L., Rubin, A.N., Stafford, A.M., Angara, K., Minasi, P., Rubenstein, J.L., Sohal, 1121 V.S., and Vogt, D. (2019). Tsc1 represses parvalbumin expression and fast-spiking properties in 1122 somatostatin lineage cortical interneurons. Nature Communications 10, 4994.

1123 Marin, O. (2012). Interneuron dysfunction in psychiatric disorders. Nat Rev Neurosci 13, 107-120.

1124 Mathis, A., Mamidanna, P., Cury, K.M., Abe, T., Murthy, V.N., Mathis, M.W., and Bethge, M. (2018). 1125 DeepLabCut: markerless pose estimation of user-defined body parts with deep learning. Nat Neurosci $112621,1281-1289$.

1127 McKenzie, S. (2018). Inhibition shapes the organization of hippocampal representations. Hippocampus 1128 28, 659-671.

1129 Melzer, S., and Monyer, H. (2020). Diversity and function of corticopetal and corticofugal GABAergic 1130 projection neurons. Nat Rev Neurosci 21, 499-515.

1131 Melzer, S., Michael, M., Caputi, A., Eliava, M., Fuchs, E.C., Whittington, M.A., and Monyer, H. (2012). 1132 Long-range-projecting GABAergic neurons modulate inhibition in hippocampus and entorhinal cortex. 1133 Science $335,1506-1510$.

1134 Miller, E.K., and Cohen, J.D. (2001). An integrative theory of prefrontal cortex function. Annu. Rev. 1135 Neurosci. 24, 167-202.

1136 Miller, E.K. (2000). The prefontral cortex and cognitive control. Nat Rev Neurosci 1, 59-65.

1137 Milstein, A.D., Bloss, E.B., Apostolides, P.F., Vaidya, S.P., Dilly, G.A., Zemelman, B.V., and Magee, 1138 J.C. (2015). Inhibitory Gating of Input Comparison in the CA1 Microcircuit. Neuron 87, 1274-1289.

1139 Moser, E.I., Kropff, E., and Moser, M.-B. (2008). Place Cells, Grid Cells, and the Brain's Spatial 1140 Representation System. Annu. Rev. Neurosci. 31, 69-89.

1141 Mukamel, E.A., Nimmerjahn, A., and Schnitzer, M.J. (2009). Automated analysis of cellular signals 1142 from large-scale calcium imaging data. Neuron 63, 747-760. 
1143 O'Keefe, J. (1976). Place units in the hippocampus of the freely moving rat. Experimental Neurology 51, $114478-109$.

1145 O'Neill, P.K., Gordon, J.A., and Sigurdsson, T. (2013). Theta Oscillations in the Medial Prefrontal 1146 Cortex Are Modulated by Spatial Working Memory and Synchronize with the Hippocampus through Its 1147 Ventral Subregion. J. Neurosci 33, 14211-14224.

1148 Orellana, G., and Slachevsky, A. (2013). Executive Functioning in Schizophrenia. Front Psychiatry 4 , 114935.

1150 Paterno, R., Casalia, M., and Baraban, S.C. (2020). Interneuron deficits in neurodevelopmental 1151 disorders: Implications for disease pathology and interneuron-based therapies. European Journal of 1152 Paediatric Neurology 24, 81-88.

1153 Place, R., Farovik, A., Brockmann, M., and Eichenbaum, H. (2016). Bidirectional prefrontal1154 hippocampal interactions support context-guided memory. Nat Neurosci 19, 992-994.

1155 Potter, G.B., Petryniak, M.A., Shevchenko, E., McKinsey, G.L., Ekker, M., and Rubenstein, J.L.R. 1156 (2009). Generation of Cre-transgenic mice using Dlx1/Dlx2 enhancers and their characterization in 1157 GABAergic interneurons. 40, 167-186.

1158 Preston, A.R., and Eichenbaum, H. (2013). Interplay of hippocampus and prefrontal cortex in memory. 1159 Current Biology 23, R764-R773.

1160 Rajasethupathy, P., Sankaran, S., Marshel, J.H., Kim, C.K., Ferenczi, E., Lee, S.Y., Berndt, A., 1161 Ramakrishnan, C., Jaffe, A., Lo, M., et al. (2015). Projections from neocortex mediate top-down control 1162 of memory retrieval. Nature 526, 653-659.

1163 Shin, J.D., and Jadhav, S.P. (2016). Multiple modes of hippocampal-prefrontal interactions in memory1164 guided behavior. Systems Neuroscience 40, 161-169.

1165 Sigurdsson, T., and Duvarci, S. (2016). Hippocampal-Prefrontal Interactions in Cognition, Behavior and 1166 Psychiatric Disease. Front. Syst. Neurosci. 9, 190-190.

1167 Sigurdsson, T., Stark, K.L., Karayiorgou, M., Gogos, J.A., and Gordon, J.A. (2010). Impaired 1168 hippocampal-prefrontal synchrony in a genetic mouse model of schizophrenia. Nature 464, $763-767$.

1169 Spellman, T., Rigotti, M., Ahmari, S.E., Fusi, S., Gogos, J.A., and Gordon, J.A. (2015). Hippocampal1170 prefrontal input supports spatial encoding in working memory. Nature 522, 309-314.

1171 Stamatakis, A.M., Schachter, M.J., Gulati, S., Zitelli, K.T., Malanowski, S., Tajik, A., Fritz, C., Trulson, 1172 M., and Otte, S.L. (2018). Simultaneous Optogenetics and Cellular Resolution Calcium Imaging During 1173 Active Behavior Using a Miniaturized Microscope. Front. Neurosci. 12, 496-496.

1174 Tamamaki, N., and Tomioka, R. (2010). Long-Range GABAergic Connections Distributed throughout 1175 the Neocortex and their Possible Function. Front. Neurosci. 4, 202.

1176 Trimper, J.B., Galloway, C.R., Jones, A.C., Mandi, K., and Manns, J.R. (2017). Gamma Oscillations in 1177 Rat Hippocampal Subregions Dentate Gyrus, CA3, CA1, and Subiculum Underlie Associative Memory 1178 Encoding. CellReports 21, 2419-2432. 
1179 Tukker, J.J., Fuentealba, P., Hartwich, K., Somogyi, P., and Klausberger, T. (2007). Cell Type-Specific 1180 Tuning of Hippocampal Interneuron Firing during Gamma Oscillations In Vivo. J Neurosci 27, 8184.

1181 Turi, G.F., Li, W.-K., Chavlis, S., Pandi, I., O'Hare, J., Priestley, J.B., Grosmark, A.D., Liao, Z., Ladow, 1182 M., Zhang, J.F., et al. (2019). Vasoactive Intestinal Polypeptide-Expressing Interneurons in the 1183 Hippocampus Support Goal-Oriented Spatial Learning. Neuron 101, 1150-1165.e1158.

1184 Vertes, R.P., Hoover, W.B., Szigeti-Buck, K., and Leranth, C. (2007). Nucleus reuniens of the midline 1185 thalamus: Link between the medial prefrontal cortex and the hippocampus. Brain Research Bulletin 71, 1186 601-609.

1187 Vinck, M., Oostenveld, R., van Wingerden, M., Battaglia, F., and Pennartz, C.M.A. (2011). An 1188 improved index of phase-synchronization for electrophysiological data in the presence of volume1189 conduction, noise and sample-size bias. Neuroimage 55, 1548-1565.

1190 Wang, G.-W., and Cai, J.-X. (2006). Disconnection of the hippocampal-prefrontal cortical circuits 1191 impairs spatial working memory performance in rats. Behavioural Brain Research 175, 329-336.

1192 Wilson, M.A., and McNaughton, B.L. (1993). Dynamics of the hippocampal ensemble code for space. 1193 Science $261,1055$.

1194 Xu, W., and Südhof, T.C. (2013). A neural circuit for memory specificity and generalization. Science $1195339,1290-1295$.

1196 Yoon, T., Okada, J., Jung, M.W., and Kim, J.J. (2008). Prefrontal cortex and hippocampus subserve 1197 different components of working memory in rats. Learn. Mem. 15, 97-105.

1198 Yu, J.Y., and Frank, L.M. (2015). Hippocampal-cortical interaction in decision making. Neurobiology 1199 of Learning and Memory 117, 34-41. 